\title{
Crystal graphs for Lie superalgebras and Cauchy decomposition
}

\author{
Jae-Hoon Kwon
}

Received: 22 November 2005 / Accepted: 1 May 2006 /

Published online: 11 July 2006

(C) Springer Science + Business Media, LLC 2007

\begin{abstract}
We discuss Cauchy type decompositions of crystal graphs for general linear Lie superalgebras. More precisely, we consider bicrystal graph structures on various sets of matrices of non-negative integers, and obtain their decompositions with explicit combinatorial isomorphisms.
\end{abstract}

Keywords Crystal graphs $\cdot$ Lie superalgebra $\cdot$ Cauchy decomposition

\section{Introduction}

Let $\mathfrak{g l}_{m \mid n}$ be the general linear Lie superalgebra over $\mathbb{C}$, and let $\mathbb{C}^{m \mid n}$ be its $(m+n)$-dimensional natural representation with the $\mathbb{Z}_{2}$-grading $\left(\mathbb{C}^{m \mid n}\right)_{0}=\mathbb{C}^{m}$ and $\left(\mathbb{C}^{m \mid n}\right)_{1}=\mathbb{C}^{n}$. A tensor power of $\mathbb{C}^{m \mid n}$ is completely reducible from Schur-Weyl duality, and its irreducible components, called irreducible polynomial representations, are parameterized by $\mathcal{P}_{m \mid n}$, the set of all $(m, n)$-hook partitions [2]. Let $S\left(\mathbb{C}^{m \mid n} \otimes \mathbb{C}^{u \mid v}\right)$ be the (super) symmetric algebra generated by the $\left(\mathfrak{g l}_{m \mid n}, \mathfrak{g l}_{u \mid v}\right)$-bimodule $\mathbb{C}^{m \mid n} \otimes \mathbb{C}^{u \mid v}$. From Howe duality, it is also completely reducible as a $\left(\mathfrak{g l}_{m \mid n}, \mathfrak{g l}_{u \mid v}\right)$-bimodule, and we have the following Cauchy type decomposition;

$$
S\left(\mathbb{C}^{m \mid n} \otimes \mathbb{C}^{u \mid v}\right)=\bigoplus_{\lambda \in \mathcal{P}_{m \mid n} \cap \mathcal{P}_{u \mid v}} V_{m \mid n}(\lambda) \otimes V_{u \mid v}(\lambda),
$$

This research was supported by 2005 research fund of University of Seoul.

J.-H. Kwon

Department of Mathematics, University of Seoul, 90 Cheonnong-dong, Dongdaemun-gu,

Seoul 130-743, Korea

e-mail: jhkwon@uos.ac.kr 
where $V_{m \mid n}(\lambda)$ and $V_{u \mid v}(\lambda)$ denote the irreducible polynomial representations of $\mathfrak{g l}_{m \mid n}$ and $\mathfrak{g l}_{u \mid v}$, respectively, corresponding to $\lambda$ (see $[4,9]$ ). In terms of characters, (1.1) also yields a Cauchy type identity of hook Schur functions (cf. $[18,19])$.

The purpose of this paper is to understand the decomposition (1.1) within a framework of (abstract) crystal graphs for Lie superalgebras which were developed by Benkart, Kang and Kashiwara [1]. For $\lambda \in \mathcal{P}_{m \mid n}$, we denote by $\mathbf{B}_{m \mid n}(\lambda)$ the set of all $(m, n)$-hook semistandard tableaux of shape $\lambda$, which parameterizes the basis element of $V_{m \mid n}(\lambda)$ [2]. According to the crystal base theory in [1], $\mathbf{B}_{m \mid n}(\lambda)$ becomes a colored oriented graph, which we call a crystal graph for $\mathfrak{g l}_{m \mid n}$ or $\mathfrak{g l}_{m \mid n}$-crystal. As in the case of symmetrizable Kac-Moody algebras, the crystal graphs for $\mathfrak{g l}_{m \mid n}$ have nice behaviors under tensor product, and we can decompose various finite dimensional representations of $\mathfrak{g l}_{m \mid n}$ in a purely combinatorial way (cf. [14]).

For non-negative integers $m, n, u, v$ such that $m+n, u+v>0$, let

$$
\mathbf{M}=\left\{A=\left(a_{b b^{\prime}}\right)_{b \in \mathbf{B}_{m \mid n}, b^{\prime} \in \mathbf{B}_{u \mid v}} \mid \text { (i) } a_{b b^{\prime}} \in \mathbb{Z}_{\geq 0} \text {, (ii) } a_{b b^{\prime}} \leq 1 \text { if }|b| \neq\left|b^{\prime}\right|\right\}
$$

where $\mathbf{B}_{m \mid n}$ (resp. $\mathbf{B}_{u \mid v}$ ) is the crystal graph associated to the natural representation of $\mathfrak{g l}_{m \mid n}\left(\right.$ resp. $\left.\mathfrak{g l}_{u \mid v}\right)$, and $|b|$ denotes the degree of $b$. Note that $\mathbf{M}$ naturally parameterizes the monomial basis of $S\left(\mathbb{C}^{m \mid n} \otimes \mathbb{C}^{u \mid v}\right)$. Then we show that as a crystal graph for $\mathfrak{g l}_{m \mid n} \oplus \mathfrak{g l}_{u \mid v}\left(\right.$ or $\left(\mathfrak{g l}_{m \mid n}, \mathfrak{g l}_{u \mid v}\right)$-bicrystal $)$,

$$
\mathbf{M} \simeq \bigoplus_{\lambda \in \mathcal{P}_{m \mid n} \cap \mathcal{P}_{u \mid v}} \mathbf{B}_{m \mid n}(\lambda) \times \mathbf{B}_{u \mid v}(\lambda)
$$

The isomorphism is given by a super-analogue of the well-known Knuth correspondence [17] (cf. [6]). But our proof is different from the original one since the decomposition is given by characterizing all the highest weight elements of the connected components from the view point of crystal graphs. Furthermore, our approach enables us to explain several variations of the Knuth correspondence (cf. [7]) in a unified way, and also derive an interesting relation between the statistics of the diagonal entries of a symmetric matrix in $\mathbf{M}$ and the number of odd parts in the shape of the corresponding tableau (a special case of this relation was first observed in [17]).

We may naturally extend the above decomposition to a semi-infinite case. Let $\mathfrak{g}$ be a contragredient Lie superalgebra of infinite rank whose Dynkin diagram is given by

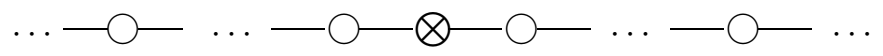

(see [11]). First, we introduce a $\mathfrak{g}$-crystal $\mathscr{F}$ consisting of semi-infinite words, which can be viewed as a crystal graph associated to a Fock space representation of $\mathfrak{g}$ analogous to the level one fermionic Fock space representation of $\widehat{\mathfrak{g l}}_{\infty}$ (cf. [10]). We show that a connected component of a tensor power $\mathscr{F}^{\otimes u}(u \geq 1)$ can be realized as the set of semi-infinite semistandard tableaux, which is generated by a highest weight element. Moreover, an explicit multiplicity-free decomposition of $\mathscr{F}^{\otimes u}$ as a $\left(\mathfrak{g}, \mathfrak{g l}_{u}\right)$-bicrystal is given, where each connected component is parameterized by a generalized partition of length $u$. To prove this, we identify $\mathscr{F} \otimes u$ with a set of Springer 
certain matrices with infinite number of rows, and apply the methods used in the case of finite ranks. More precisely, an element in $\mathscr{F}^{\otimes u}$ is equivalent to a unique pair of a semi-infinite semistandard tableau and a rational semistandard tableau as an element in a $\left(\mathfrak{g}, \mathfrak{g l}_{u}\right)$-bicrystal. Hence, it gives rise to a Knuth correspondence of a semi-infinite type, and a Cauchy type identity. As a by-product, we obtain a character formula of a $\mathfrak{g}$-crystal of semi-infinite semistandard tableaux occurring as a connected component in $\mathscr{F}^{\otimes u}$. This character formula is given in terms of ordinary Schur functions and the Littlewood-Richardson coefficients, and it is very similar to the ones of the irreducible highest weight representations of $\widehat{\mathfrak{g l}}_{\infty}$ or $\widehat{\mathfrak{g l}}_{\infty \mid \infty}$ obtained in $[3,13]$. In fact, using crystal graphs, we can give a similar combinatorial proof of the Cauchy type decomposition of a higher level fermionic Fock space representation of $\widehat{\mathfrak{g l}}_{\infty}$ given by Kac and Radul [13], and hence the character formula of irreducible highest weight representations of $\widehat{\mathfrak{g l}}_{\infty}$. We also expect a combinatorial proof of the decomposition of a Fock space representation of $\mathfrak{g l}_{\infty \mid \infty}$ given by Cheng and Wang [5].

The paper is organized as follows. In Section 2, we review the basic notions and the main results on crystal graphs for $\mathfrak{g l}_{m \mid n}$ in [1]. In Section 3, we prove the decomposition (1.2) and then study the diagonal action of the Kashiwara operators on the set of symmetric matrices in M. In Section 4, we describe the dual decomposition which is associated to the (super) exterior algebra $\Lambda\left(\mathbb{C}^{m \mid n} \otimes \mathbb{C}^{u \mid v}\right)$. Finally, in Section 5, we introduce a $\mathfrak{g}$-crystal of semi-infinite semistandard tableaux, and a $\mathfrak{g l}_{u}$-crystal of rational semistandard tableaux (cf. [20]). Then using these combinatorial realizations of crystal graphs, we establish a Cauchy type decomposition of a tensor power $\mathscr{F}^{\otimes u}$ as a $\left(\mathfrak{g}, \mathfrak{g l}_{u}\right)$-bicrystal.

\section{Crystal graphs for $\mathfrak{g l}_{m \mid n}$}

In this section, we recall the basic notions on crystal graphs for $\mathfrak{g l}_{m \mid n}$ developed in [1].

\subsection{Definitions}

For non-negative integers $m, n$ with $m+n>0$, let $\mathfrak{g l}_{m \mid n}$ be the general linear Lie superalgebra over $\mathbb{C}$ (see [11]). Let

$$
\mathbf{B}_{m \mid n}=\{\bar{m}<\overline{m-1}<\cdots<\overline{1}<1<2<\cdots<n\}
$$

be a linearly ordered set. Set $\mathbf{B}_{m \mid n}^{+}=\{\bar{m}, \overline{m-1}, \ldots, \overline{1}\}$ and $\mathbf{B}_{m \mid n}^{-}=\{1,2, \ldots, n\}$. For $b \in \mathbf{B}_{m \mid n}$, we define $|b|$, degree of $b$, by $|b|=0$ (resp. 1) if $b \in \mathbf{B}_{m \mid n}^{+}\left(\operatorname{resp} . \mathbf{B}_{m \mid n}^{-}\right.$). The free abelian group $P_{m \mid n}=\bigoplus_{b \in \mathbf{B}_{m \mid n}} \mathbb{Z} \epsilon_{b}$, which is generated by $\epsilon_{b}\left(b \in \mathbf{B}_{m \mid n}\right)$, is called the weight lattice of $\mathfrak{g l}_{m \mid n}$. There is a natural symmetric $\mathbb{Z}$-bilinear form (, ) on $P_{m \mid n}$, where $\left(\epsilon_{b}, \epsilon_{b^{\prime}}\right)=(-1)^{|b|} \delta_{b b^{\prime}}$ for $b, b^{\prime} \in \mathbf{B}_{m \mid n}$. Let

$$
I_{m \mid n}=\{\overline{m-1}, \ldots, \overline{1}, 0,1, \ldots, n-1\} .
$$


The simple root $\alpha_{i}\left(i \in I_{m \mid n}\right)$ of $\mathfrak{g l}_{m \mid n}$ is given by

$$
\begin{cases}\alpha_{\bar{k}}=\epsilon_{\overline{k+1}}-\epsilon_{\bar{k}}, & \text { for } k=1, \ldots, m-1, \\ \alpha_{l}=\epsilon_{l}-\epsilon_{l+1}, & \text { for } l=1, \ldots, n-1, \\ \alpha_{0}=\epsilon_{\overline{1}}-\epsilon_{1} . & \end{cases}
$$

Set $Q=\bigoplus_{i \in I_{m \mid n}} \mathbb{Z} \alpha_{i}$, which we call the root lattice of $\mathfrak{g l}_{m \mid n}$. A partial ordering on $P_{m \mid n}$ is given by $\lambda \geq \mu$ if and only if $\lambda-\mu \in \sum_{i \in I_{m \mid n}} \mathbb{Z}_{\geq 0} \alpha_{i}$ for $\lambda, \mu \in P_{m \mid n}$. We also define the simple coroot $h_{i} \in P_{m \mid n}^{*}\left(i \in I_{m \mid n}\right)$ by

$$
\left\langle h_{i}, \lambda\right\rangle= \begin{cases}\left(\alpha_{i}, \lambda\right), & \text { if } i=\overline{m-1}, \ldots, \overline{1}, 0 \\ -\left(\alpha_{i}, \lambda\right), & \text { if } i=1, \ldots, n-1\end{cases}
$$

for $\lambda \in P_{m \mid n}$, where $\langle$,$\rangle is the natural pairing on P_{m \mid n}^{*} \times P_{m \mid n}$. With respect to the above simple roots, the Dynkin diagram is

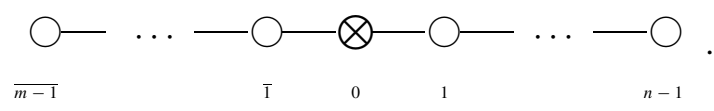

Motivated by the crystal bases of integral representations of the quantized enveloping algebra $U_{q}\left(\mathfrak{g l}_{m \mid n}\right)$, we introduce the notion of abstract crystal graphs for $\mathfrak{g l}_{m \mid n}$.

Definition 2.1. (1) A crystal graph for $\mathfrak{g l}_{m \mid n}$ (or $\mathfrak{g l}_{m \mid n}$-crystal) is a set $B$ together with the maps

$$
\begin{gathered}
\text { wt }: B \rightarrow P_{m \mid n}, \\
\varepsilon_{i}, \varphi_{i}: B \rightarrow \mathbb{Z}_{\geq 0}, \\
e_{i}, f_{i}: B \rightarrow B \cup\{\boldsymbol{0}\},
\end{gathered}
$$

for $i \in I_{m \mid n}(\mathbf{0}$ is a formal symbol), satisfying the following conditions;

(a) for $i \in I_{m \mid n}$ and $b \in B$, we have

$$
\begin{aligned}
& \varphi_{i}(b)-\varepsilon_{i}(b)=\left\langle h_{i}, \operatorname{wt}(b)\right\rangle, \quad(i \neq 0), \\
& \left\langle h_{0}, \operatorname{wt}(b)\right\rangle \geq 0, \text { and } \varphi_{0}(b)+\varepsilon_{0}(b)= \begin{cases}0, & \text { if }\left\langle h_{0}, \operatorname{wt}(b)\right\rangle=0, \\
1, & \text { if }\left\langle h_{0}, \operatorname{wt}(b)\right\rangle>0,\end{cases}
\end{aligned}
$$

(b) if $e_{i} b \in B$ for $i \in I_{m \mid n}$ and $b \in B$, then

$$
\varepsilon_{i}\left(e_{i} b\right)=\varepsilon_{i}(b)-1, \quad \varphi_{i}\left(e_{i} b\right)=\varphi_{i}(b)+1, \quad \operatorname{wt}\left(e_{i} b\right)=\operatorname{wt}(b)+\alpha_{i},
$$

(c) if $f_{i} b \in B$ for $i \in I_{m \mid n}$ and $b \in B$, then

$$
\varepsilon_{i}\left(f_{i} b\right)=\varepsilon_{i}(b)+1, \quad \varphi_{i}\left(f_{i} b\right)=\varphi_{i}(b)-1, \quad \operatorname{wt}\left(f_{i} b\right)=\operatorname{wt}(b)-\alpha_{i},
$$


(d) $f_{i} b=b^{\prime}$ if and only if $b=e_{i} b^{\prime}$ for all $i \in I_{m \mid n}, b, b^{\prime} \in B$,

(We call $e_{i}$ and $f_{i}\left(i \in I_{m \mid n}\right)$ the Kashiwara operators).

(2) Let $B$ be a crystal graph for $\mathfrak{g l}_{m \mid n}$. A subset $B^{\prime} \subset B$ is called a subcrystal of $B$ if $B^{\prime}$ is itself a crystal graph for $\mathfrak{g l}_{m \mid n}$ with respect to wt, $\varepsilon_{i}, \varphi_{i}, e_{i}, f_{i}\left(i \in I_{m \mid n}\right)$ of $B$.

Remark 2.2. (1) The above definition is based on the crystal bases of integrable representations of $U_{q}\left(\mathfrak{g l}_{m \mid n}\right)$ in [1], while crystal graphs for contragredient Lie superalgebras might be defined in a more general sense, as in the case of symmetrizable Kac-Moody algebras (cf. [15, 16]).

(2) A crystal graph $B$ becomes an $I_{m \mid n}$-colored oriented graph, where

$$
b \stackrel{i}{\rightarrow} b^{\prime} \quad \text { if and only if } \quad b^{\prime}=f_{i} b \quad\left(i \in I_{m \mid n}\right)
$$

Definition 2.3. Let $B_{1}$ and $B_{2}$ be crystal graphs for $\mathfrak{g l}_{m \mid n}$. We define the tensor product of $B_{1}$ and $B_{2}$ to be the set $B_{1} \otimes B_{2}=\left\{b_{1} \otimes b_{2} \mid b_{i} \in B_{i},(i=1,2)\right\}$ with

$$
\begin{gathered}
\operatorname{wt}\left(b_{1} \otimes b_{2}\right)=\operatorname{wt}\left(b_{1}\right)+\operatorname{wt}\left(b_{2}\right), \\
\varepsilon_{i}\left(b_{1} \otimes b_{2}\right)= \begin{cases}\max \left(\varepsilon_{i}\left(b_{1}\right), \varepsilon_{i}\left(b_{2}\right)-\left\langle h_{i}, \operatorname{wt}\left(b_{1}\right)\right\rangle\right), & \text { if } i=\overline{m-1}, \ldots, \overline{1}, \\
\max \left(\varepsilon_{i}\left(b_{1}\right)-\left\langle h_{i}, \operatorname{wt}\left(b_{2}\right)\right\rangle, \varepsilon_{i}\left(b_{2}\right)\right), & \text { if } i=1, \ldots, n-1, \\
\varepsilon_{0}\left(b_{1}\right), & \text { if } i=0,\left\langle h_{0}, \operatorname{wt}\left(b_{1}\right)\right\rangle>0, \\
\varepsilon_{0}\left(b_{2}\right), & \text { if } i=0,\left\langle h_{0}, \operatorname{wt}\left(b_{1}\right)\right\rangle=0,\end{cases} \\
\varphi_{i}\left(b_{1} \otimes b_{2}\right)= \begin{cases}\max \left(\varphi_{i}\left(b_{1}\right)+\left\langle h_{i}, \operatorname{wt}\left(b_{2}\right)\right\rangle, \varphi_{i}\left(b_{2}\right)\right), & \text { if } i=\overline{m-1}, \ldots, \overline{1}, \\
\max \left(\varphi_{i}\left(b_{1}\right), \varphi_{i}\left(b_{2}\right)+\left\langle h_{i}, \operatorname{wt}\left(b_{1}\right)\right\rangle\right), & \text { if } i=1, \ldots, n-1, \\
\varphi_{0}\left(b_{1}\right), & \text { if } i=0,\left\langle h_{0}, \operatorname{wt}\left(b_{1}\right)\right\rangle>0, \\
\varphi_{0}\left(b_{2}\right), & \text { if } i=0,\left\langle h_{0}, \operatorname{wt}\left(b_{1}\right)\right\rangle=0,\end{cases}
\end{gathered}
$$$$
e_{i}\left(b_{1} \otimes b_{2}\right)= \begin{cases}e_{i} b_{1} \otimes b_{2}, & \text { if } i=\overline{m-1}, \ldots, \overline{1}, \varphi_{i}\left(b_{1}\right) \geq \varepsilon_{i}\left(b_{2}\right), \\ b_{1} \otimes e_{i} b_{2}, & \text { if } i=\overline{m-1}, \ldots, \overline{1}, \varphi_{i}\left(b_{1}\right)<\varepsilon_{i}\left(b_{2}\right), \\ e_{i} b_{1} \otimes b_{2}, & \text { if } i=1, \ldots, n-1, \varphi_{i}\left(b_{2}\right)<\varepsilon_{i}\left(b_{1}\right), \\ b_{1} \otimes e_{i} b_{2}, & \text { if } i=1, \ldots, n-1, \varphi_{i}\left(b_{2}\right) \geq \varepsilon_{i}\left(b_{1}\right), \\ e_{0} b_{1} \otimes b_{2}, & \text { if } i=0,\left\langle h_{0}, \operatorname{wt}\left(b_{1}\right)\right\rangle>0, \\ b_{1} \otimes e_{0} b_{2}, & \text { if } i=0,\left\langle h_{0}, \operatorname{wt}\left(b_{1}\right)\right\rangle=0,\end{cases}
$$ 


$$
f_{i}\left(b_{1} \otimes b_{2}\right)= \begin{cases}f_{i} b_{1} \otimes b_{2}, & \text { if } i=\overline{m-1}, \ldots, \overline{1}, \varphi_{i}\left(b_{1}\right)>\varepsilon_{i}\left(b_{2}\right), \\ b_{1} \otimes f_{i} b_{2}, & \text { if } i=\overline{m-1}, \ldots, \overline{1}, \varphi_{i}\left(b_{1}\right) \leq \varepsilon_{i}\left(b_{2}\right), \\ f_{i} b_{1} \otimes b_{2}, & \text { if } i=1, \ldots, n-1, \varphi_{i}\left(b_{2}\right) \leq \varepsilon_{i}\left(b_{1}\right), \\ b_{1} \otimes f_{i} b_{2}, & \text { if } i=1, \ldots, n-1, \varphi_{i}\left(b_{2}\right)>\varepsilon_{i}\left(b_{1}\right), \\ f_{0} b_{1} \otimes b_{2}, & \text { if } i=0,\left\langle h_{0}, \operatorname{wt}\left(b_{1}\right)\right\rangle>0, \\ b_{1} \otimes f_{0} b_{2}, & \text { if } i=0,\left\langle h_{0}, \operatorname{wt}\left(b_{1}\right)\right\rangle=0,\end{cases}
$$

where we assume that $\mathbf{0} \otimes b_{2}=b_{1} \otimes \mathbf{0}=\mathbf{0}$.

Then, it is straightforward to check that $B_{1} \otimes B_{2}$ is a crystal graph for $\mathfrak{g l}_{m \mid n}$.

Definition 2.4. Let $B_{1}$ and $B_{2}$ be two crystal graphs for $\mathfrak{g l}_{m \mid n}$.

(1) The direct sum $B_{1} \oplus B_{2}$ is the disjoint union of $B_{1}$ and $B_{2}$.

(2) An isomorphism $\psi: B_{1} \rightarrow B_{2}$ of $\mathfrak{g l}_{m \mid n}$-crystals is an isomorphism of $I_{m \mid n}$-colored oriented graphs which preserves wt, $\varepsilon_{i}$, and $\varphi_{i}\left(i \in I_{m \mid n}\right)$. We say that $B_{1}$ is isomorphic to $B_{2}$, and write $B_{1} \simeq B_{2}$.

(3) For $b_{i} \in B_{i}(i=1,2)$, let $C\left(b_{i}\right)$ denote the connected component of $b_{i}$ as an $I_{m \mid n^{-}}$ colored oriented graph. We say that $b_{1}$ is $\mathfrak{g l}_{m \mid n}$-equivalent to $b_{2}$, if there is an isomorphism of crystal graphs $C\left(b_{1}\right) \rightarrow C\left(b_{2}\right)$ sending $b_{1}$ to $b_{2}$. We often write $b_{1} \simeq_{\mathfrak{g l}_{m \mid n}} b_{2}$ (or simply $b_{1} \simeq b_{2}$ if there is no confusion).

\subsection{Semistandard tableaux for $\mathfrak{g l}_{m \mid n}$}

$\mathbf{B}_{m \mid n}$ becomes a crystal graph for $\mathfrak{g l}_{m \mid n}$ whose associated $I_{m \mid n}$-colored oriented graph is given by

$$
\bar{m} \stackrel{\overline{m-1}}{\longrightarrow} \overline{m-1} \stackrel{\overline{m-2}}{\longrightarrow} \cdots \stackrel{\overline{1}}{\longrightarrow} \overline{1} \stackrel{0}{\longrightarrow} 1 \stackrel{1}{\longrightarrow} \cdots \stackrel{n-2}{\longrightarrow} n-1 \stackrel{n-1}{\longrightarrow} n,
$$

where $\operatorname{wt}(b)=\epsilon_{b}$, and $\varepsilon_{i}(b)$ (resp. $\left.\varphi_{i}(b)\right)$ is the number of $i$-colored arrows coming into $b$ (resp. going out of $b$ ) for $i \in I_{m \mid n}$ and $b \in \mathbf{B}_{m \mid n}$. Note that $\mathbf{B}_{m \mid n}$ is the crystal graph associated to the natural representation $\mathbb{C}^{m \mid n}$.

Let $\mathcal{W}_{m \mid n}$ be the set of all finite words with the letters in $\mathbf{B}_{m \mid n}$. The empty word is denoted by $\emptyset$. Then $\mathcal{W}_{m \mid n}$ is a crystal graph for $\mathfrak{g l}_{m \mid n}$ since we may identify each non-empty word $w=w_{1} \ldots w_{r}$ with $w_{1} \otimes \cdots \otimes w_{r} \in \mathbf{B}_{m \mid n}^{\otimes r}$, where $\{\emptyset\}$ forms a trivial crystal graph, that is, $\operatorname{wt}(\emptyset)=0, e_{i} \emptyset=f_{i} \emptyset=\mathbf{0}$, and $\varepsilon_{i}(\emptyset)=\varphi_{i}(\emptyset)=0$ for all $i \in I_{m \mid n}$.

Following the tensor product rule in Definition 2.3, we can describe the Kashiwara operators $e_{i}, f_{i}: \mathcal{W}_{m \mid n} \rightarrow \mathcal{W}_{m \mid n} \cup\{\mathbf{0}\}\left(i \in I_{m \mid n}\right)$ in a more explicit way;

(1) Suppose that a non-empty word $w=w_{1} \ldots w_{r}$ is given. To each letter $w_{k}$, we assign

$$
\epsilon^{(i)}\left(w_{k}\right)= \begin{cases}+, & \text { if }\left(i=\bar{p}, w_{k}=\overline{p+1}\right), \text { or }\left(i=p, w_{k}=p\right), \text { or }\left(i=0, w_{k}=\overline{1}\right), \\ -, & \text { if }\left(i=\bar{p}, w_{k}=\bar{p}\right), \text { or }\left(i=p, w_{k}=p+1\right), \text { or }\left(i=0, w_{k}=1\right), \\ \cdot, & \text { otherwise }\end{cases}
$$

and let $\epsilon^{(i)}(w)=\left(\epsilon^{(i)}\left(w_{1}\right), \ldots, \epsilon^{(i)}\left(w_{r}\right)\right)$. 
(2) If $i=\bar{p}$ for $1 \leq p \leq m-1$, then we replace a pair $\left(\epsilon^{(i)}\left(w_{s}\right), \epsilon^{(i)}\left(w_{s^{\prime}}\right)\right)=(+,-)$ such that $s<s^{\prime}$ and $\epsilon^{(i)}\left(w_{t}\right)=\cdot$ for $s<t<s^{\prime}$ by $(\cdot, \cdot)$ in $\epsilon^{(i)}(w)$, and repeat this process as far as possible until we get a sequence with no + placed to the left of - . If $i=p$ for $1 \leq p \leq n-1$, then we do the same work for $(-,+)$-pair in $\epsilon^{(i)}(w)$ until we get a sequence with no - placed to the left of + . We call this sequence the $i$-signature of $w$. If $i=0$, then we define the 0-signature of $w$ to be $\epsilon^{(i)}\left(w_{k}\right)(\neq \cdot)$ such that $\epsilon^{(i)}\left(w_{l}\right)=\cdot$ for all $1 \leq l<k$.

(3) If $i=\bar{p}$ (resp. $i=p$ ), then we call the right-most (resp. left-most) - in the $i$ signature of $w$ the $i$-good - sign, and define $e_{i} w$ to be the word obtained by applying $e_{i}$ to $\bar{p}$ (resp. $p+1$ ) corresponding to the $i$-good - sign. If there is no $i$-good - sign, then we define $e_{i} w=\mathbf{0}$.

(4) If $i=\bar{p}$ (resp. $i=p$ ), then we call the left-most (resp. right-most) + in the $i$ signature of $w$ the $i$-good + sign, and define $f_{i} w$ to be the word obtained by applying $f_{i}$ to $\overline{p+1}$ (resp. $p$ ) corresponding to the $i$-good + sign. If there is no $i$-good + sign, then we define $f_{i} w=\mathbf{0}$.

(5) If $i=0$, then we define $e_{0} w$ (resp. $f_{0} w$ ) to be the word obtained by applying $e_{0}$ (resp. $f_{0}$ ) to the letter corresponding to the 0 -signature of $w$. If the 0 -signature of $w$ is empty, then we define $e_{0} w=\mathbf{0}$ (resp. $f_{0} w=\mathbf{0}$ ).

Note that we have

$$
\varepsilon_{i}(w)=\max \left\{k \mid e_{i}^{k} w \neq \mathbf{0}\right\}, \quad \varphi_{i}(w)=\max \left\{k \mid f_{i}^{k} w \neq \mathbf{0}\right\}
$$

for $w \in \mathcal{W}_{m \mid n}$ and $i \in I_{m \mid n}$.

Example 2.5. Suppose that

$$
w=1 \overline{1} \overline{1} \overline{1} \overline{2} 22 \overline{2} 1 \text {. }
$$

Then

$$
\begin{aligned}
& \epsilon^{(\overline{1})}(w)=(\cdot,-,-\ominus, \oplus, \cdot, \cdot,+\cdot \cdot), \\
& \epsilon^{(1)}(w)=(\oplus, \cdot, \cdot, \cdot \cdot, \ominus,-, \cdot,+), \\
& \epsilon^{(0)}(w)=(\ominus,+,+,+, \cdot, \cdot, \cdot, \cdot,-),
\end{aligned}
$$

where $\oplus, \ominus$ denote the $i$-good signs $(i \neq 0)$, or the 0 -signature. We have

$$
\begin{array}{ll}
e_{\overline{1}}(w)=1 \overline{1} \overline{1} \overline{2} 222 \overline{2} 1, & f_{\overline{1}}(w)=1 \overline{1} \overline{1} \overline{1} 122 \overline{2} 1, \\
e_{1}(w)=1 \overline{1} \overline{1} \overline{1} 212 \overline{2} 1, & f_{1}(w)=2 \overline{1} \overline{1} \overline{1} 222 \overline{2} 1, \\
e_{0}(w)=\overline{1} \overline{1} \overline{1} \overline{1} 22 \overline{2} 1, & f_{0}(w)=\mathbf{0} .
\end{array}
$$

A partition is a non-increasing sequence of non-negative integers $\lambda=\left(\lambda_{k}\right)_{k \geq 1}$ such that all but a finite number of its terms are zero. Each $\lambda_{k}$ is called a part of $\lambda$, and the number of non-zero parts is called the length of $\lambda$. We also write $\lambda=\left(1^{m_{1}}, 2^{m_{2}}, \ldots\right)$ 
where $m_{i}$ is the number of occurrences of $i$ in $\lambda$. Recall that a partition $\lambda=\left(\lambda_{k}\right)_{k \geq 1}$ is identified with a Young diagram which is a collection of nodes (or boxes) in leftjustified rows with $\lambda_{k}$ nodes in the $k^{\text {th }}$ row.

A partition $\lambda=\left(\lambda_{k}\right)_{k \geq 1}$ is called an $(m, n)$-hook partition if $\lambda_{m+1} \leq n$. We denote by $\mathcal{P}_{m \mid n}$ the set of all $(m, n)$-hook partitions. A tableau $T$ obtained by filling a Young diagram $\lambda$ with the entries in $\mathbf{B}_{m \mid n}$ is called $(m, n)$-hook semistandard if

(1) the entries in each row (resp. column) are weakly increasing from left to right (resp. from top to bottom),

(2) the entries in $\mathbf{B}_{m \mid n}^{+}$(resp. $\mathbf{B}_{m \mid n}^{-}$) are strictly increasing in each column (resp. row)

(see [2]). We say that $\lambda$ is the shape of $T$. It is easy to see that a partition $\lambda$ can be made into an $(m, n)$-hook semistandard tableau if and only if $\lambda \in \mathcal{P}_{m \mid n}$.

For $\lambda \in \mathcal{P}_{m \mid n}$, let $\mathbf{B}_{m \mid n}(\lambda)$ be the set of all $(m, n)$-hook semistandard tableaux of shape $\lambda$. We may view $\mathbf{B}_{m \mid n}(\lambda)$ as a subset of $\mathcal{W}_{m \mid n}$ by column reading (or far eastern reading). That is, we read the entries of a tableau column by column from right to left, and in each column we read the entries from top to bottom.

For $T \in \mathbf{B}_{m \mid n}(\lambda)$, the weight of $T$ is given by wt $T=\sum_{b \in \mathbf{B}_{m \mid n}} \mu_{b} \epsilon_{b} \in P_{m \mid n}$, where $\mu_{b}$ is number of occurrences of $b$ in $T$. Indeed, $\mathbf{B}_{m \mid n}(\lambda)$ together with $\mathbf{0}$ is stable under $e_{i}, f_{i}\left(i \in I_{m \mid n}\right)$, and $\mathbf{B}_{m \mid n}(\lambda)$ is a subcrystal of $\mathcal{W}_{m \mid n}$. The following is one of the main results in [1] (see also Remark 2.7).

Theorem 2.6 ([1]). For $\lambda \in \mathcal{P}_{m \mid n}, \mathbf{B}_{m \mid n}(\lambda)$ is a crystal graph for $\mathfrak{g l}_{m \mid n}$. Moreover, $\mathbf{B}_{m \mid n}(\lambda)$ is a connected $I_{m \mid n}$-colored oriented graph with a unique highest weight element $H_{m \mid n}^{\lambda}$.

Remark 2.7. Note that $\operatorname{wt}\left(H_{m \mid n}^{\lambda}\right) \geq \operatorname{wt}(T)$ for all $T \in \mathbf{B}_{m \mid n}(\lambda)$ ([1]) (see Fig. 1), and hence $e_{i} H_{m \mid n}^{\lambda}=\mathbf{0}$ for all $i \in I_{m \mid n}$. But, unlike the crystal graphs associated to integrable highest weight representations of $U_{q}\left(\mathfrak{g l}_{n}\right)$ (in our notation, $U_{q}\left(\mathfrak{g l}_{n \mid 0}\right)$ ), there might exist $T \in \mathbf{B}_{m \mid n}(\lambda)$ such that $T \neq H_{m \mid n}^{\lambda}$ and $e_{i} T=\mathbf{0}$ for all $i \in I_{m \mid n}$. Such a tableau $T$ was called a fake highest weight vector, and $H_{m \mid n}^{\lambda}$ a genuine highest weight vector in [1]. Therefore, a given $(m, n)$-hook semistandard tableau $T$ of shape $\lambda$ is not necessarily

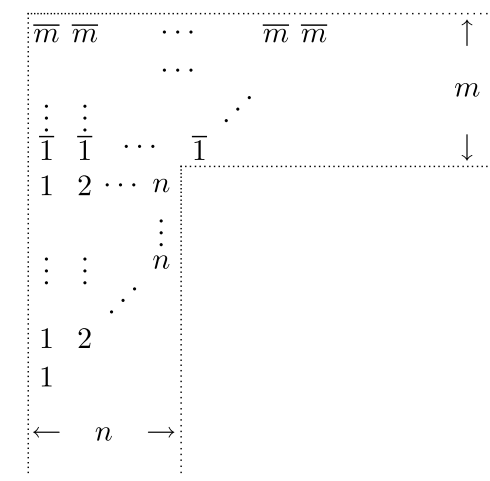

Fig. 1 A highest weight tableau $H_{m \mid n}^{\lambda}$ 
obtained from $H_{m \mid n}^{\lambda}$ by applying the operators $f_{i}$, and this fact gives rise to a special difficulty in several arguments including the proof of the connectedness of $\mathbf{B}_{m \mid n}(\lambda)$ in Theorem 2.6.

To characterize a connected component in $\mathcal{W}_{m \mid n}$, we need the algorithm of Schensted's column bumping for $(m, n)$-hook semistandard tableaux $([2,19])$ : for $\lambda \in \mathcal{P}_{m \mid n}$ and $T \in \mathbf{B}_{m \mid n}(\lambda)$, we define $T \leftarrow b\left(b \in \mathbf{B}_{m \mid n}\right)$ to be the tableau obtained from $T$ by applying the following procedure;

(1) If $b \in \mathbf{B}_{m \mid n}^{+}$, let $b^{\prime}$ be the smallest entry in the first (or the left-most) column which is greater than or equal to $b$. If $b \in \mathbf{B}_{m \mid n}^{-}$, let $b^{\prime}$ be the smallest entry in the first column which is greater than $b$. If there are more than one $b^{\prime}$, choose the one in the highest position.

(2) Replace $b^{\prime}$ by $b$ ( $b^{\prime}$ is bumped out of the first column). If there is no such $b^{\prime}$, put $b$ at the bottom of the first column and stop the procedure.

(3) Repeat (1) and (2) on the next column with $b^{\prime}$.

Note that $(T \leftarrow b) \in \mathbf{B}_{m \mid n}(\mu)$ for some $\mu \in \mathcal{P}_{m \mid n}$, where $\mu$ is given by adding a node at $\lambda$. Now, for a given word $w=w_{1} \cdots w_{r} \in \mathcal{W}$, we define

$$
P(w)=\left(\cdots\left(\left(w_{1} \leftarrow w_{2}\right) \leftarrow w_{3}\right) \cdots\right) \leftarrow w_{r} .
$$

Lemma 2.8 ([1]). For $w \in \mathcal{W}_{m \mid n}$, we have $w \simeq P(w)$. Hence, any connected component in $\mathcal{W}_{m \mid n}$ is isomorphic to $\mathbf{B}_{m \mid n}(\lambda)$ for some $\lambda \in \mathcal{P}_{m \mid n}$.

We will also use the following lemma in the next section.

Lemma 2.9 ([14]). Let $T$ and $T^{\prime}$ be two $(m, n)$-hook semistandard tableaux. If $T \simeq$ $T^{\prime}$, then $T=T^{\prime}$.

Let $x=\left\{x_{b} \mid b \in \mathbf{B}_{m \mid n}\right\}$ be the set of variables indexed by $\mathbf{B}_{m \mid n}$. For $\mu=$ $\sum_{b \in \mathbf{B}_{m \mid n}} \mu_{b} \epsilon_{b} \in P_{m \mid n}$, we set $x^{\mu}=\prod_{b \in \mathbf{B}_{m \mid n}} x_{b}^{\mu_{b}}$. For $\lambda \in \mathcal{P}_{m \mid n}$, we define a hook Schur function corresponding to $\lambda$ by

$$
h s_{\lambda}(x)=\sum_{T \in \mathbf{B}_{m \mid n}(\lambda)} x^{\mathrm{wt} T},
$$

which is the character of $\mathbf{B}_{m \mid n}(\lambda)$ (see $\left.[2,19]\right)$.

\section{Bicrystal graphs and Cauchy decomposition}

We consider a bicrystal graph structure on the set of certain matrices of non-negative integers, which parameterizes the monomial basis of $S\left(\mathbb{C}^{m \mid n} \otimes \mathbb{C}^{u \mid v}\right)$, and then derive an explicit decomposition by finding all the highest weight elements. 


\subsection{Crystal graphs of biwords}

Suppose that $m, n, u, v$ are non-negative integers such that $m+n, u+v>0$.

Let

$$
\begin{aligned}
\Omega_{m|n, u| v}= & \left\{(\mathbf{i}, \mathbf{j}) \in \mathcal{W}_{m \mid n} \times \mathcal{W}_{u \mid v} \mid\right. \\
& \text { (1) } \mathbf{i}=i_{1}, \ldots, i_{r} \text { and } \mathbf{j}=j_{1}, \ldots, j_{r} \text { for some } r \geq 0, \\
& \text { (2) }\left(i_{1}, j_{1}\right) \leq \cdots \leq\left(i_{r}, j_{r}\right), \\
& \text { (3) } \left.\left|i_{k}\right| \neq\left|j_{k}\right| \operatorname{implies}\left(i_{k}, j_{k}\right) \neq\left(i_{k \pm 1}, j_{k \pm 1}\right)\right\},
\end{aligned}
$$

where for $(i, j)$ and $(k, l) \in \mathbf{B}_{m \mid n} \times \mathbf{B}_{u \mid v}$, the super lexicographic ordering is given by

$$
(i, j)<(k, l) \Leftrightarrow \begin{cases}(j<l) & \text { or, } \\ \left(j=l \in \mathbf{B}_{u \mid v}^{+}, \text {and } i>k\right) & \text { or, } \\ \left(j=l \in \mathbf{B}_{u \mid v}^{-}, \text {and } i<k\right) . & \end{cases}
$$

Next, let $\Omega_{m|n, u| v}^{*}$ be the set of pairs $(\mathbf{k}, \mathbf{l}) \in \mathcal{W}_{m \mid n} \times \mathcal{W}_{u \mid v}$ such that $(\mathbf{l}, \mathbf{k}) \in \Omega_{u|v, m| n}$. For simplicity, we write $\Omega=\Omega_{m|n, u| v}$ and $\Omega^{*}=\Omega_{m|n, u| v}^{*}$.

Now, for $i \in I_{m \mid n}$ and $(\mathbf{i}, \mathbf{j}) \in \Omega$, we define

$$
e_{i}(\mathbf{i}, \mathbf{j})=\left(e_{i} \mathbf{i}, \mathbf{j}\right), \quad f_{i}(\mathbf{i}, \mathbf{j})=\left(f_{i} \mathbf{i}, \mathbf{j}\right),
$$

where we assume that $x_{i}(\mathbf{i}, \mathbf{j})=\mathbf{0}$ if $x_{i} \mathbf{i}=\mathbf{0}(x=e, f)$. We set $\operatorname{wt}(\mathbf{i}, \mathbf{j})=\operatorname{wt}(\mathbf{i})$, $\varepsilon_{i}(\mathbf{i}, \mathbf{j})=\varepsilon_{i}(\mathbf{i})$ and $\varphi_{i}(\mathbf{i}, \mathbf{j})=\varphi_{i}(\mathbf{i})\left(i \in I_{m \mid n}\right)$.

Similarly, for $i \in I_{u \mid v}$ and $(\mathbf{k}, \mathbf{l}) \in \Omega^{*}$, we define

$$
e_{j}^{*}(\mathbf{k}, \mathbf{l})=\left(\mathbf{k}, e_{j} \mathbf{l}\right), \quad f_{j}^{*}(\mathbf{k}, \mathbf{l})=\left(\mathbf{k}, f_{j} \mathbf{l}\right),
$$

and set $\mathrm{wt}^{*}(\mathbf{k}, \mathbf{l})=\mathrm{wt}(\mathbf{l}), \varepsilon_{j}^{*}(\mathbf{k}, \mathbf{l})=\varepsilon_{j}(\mathbf{l})$ and $\varphi_{j}^{*}(\mathbf{k}, \mathbf{1})=\varphi_{j}(\mathbf{1})\left(j \in I_{u \mid v}\right)$.

Lemma 3.1. Under the above hypothesis,

(1) the set $\Omega$ together with wt, $e_{i}, f_{i}, \varepsilon_{i}, \varphi_{i}\left(i \in I_{m \mid n}\right)$ is a crystal graph for $\mathfrak{g l}_{m \mid n}$, (2) the set $\Omega^{*}$ together with $\mathrm{wt}^{*}, e_{j}^{*}, f_{j}^{*}, \varepsilon_{j}^{*}, \varphi_{j}^{*}\left(j \in I_{u \mid v}\right)$ is a crystal graph for $\mathfrak{g l}_{u \mid v}$.

Proof: We will prove only (1) since the proof of (2) is the same. Suppose that $(\mathbf{i}, \mathbf{j}) \in$ $\Omega \backslash\{(\emptyset, \emptyset)\}$ is given where $\mathbf{i}=i_{1} \cdots i_{r}$ and $\mathbf{j}=j_{1} \ldots j_{r}$ for some $r \geq 1$. We write

$$
\mathbf{i}=\mathbf{i}_{\bar{u}} \mathbf{i}_{u-1} \cdots \mathbf{i}_{v-1} \mathbf{i}_{v},
$$

where $\mathbf{i}_{b}=i_{t_{1}} \ldots i_{t_{b}}\left(b \in \mathbf{B}_{u \mid v}\right)$ is a subword of $\mathbf{i}$ such that $j_{t_{1}}=\cdots=j_{t_{b}}=b$. 
Then, $\mathbf{i}_{b}\left(b \in \mathbf{B}_{u \mid v}\right)$ is $\mathfrak{g l}_{m \mid n}$-equivalent to an $(m, n)$-hook semistandard tableau $T_{b}$ of a single row or a single column as follows;

$$
T_{b}=\left\{\begin{array}{cl}
i_{t_{1}} \cdots i_{t_{b}} \in \mathbf{B}_{m \mid n}\left(\left(t_{b}\right)\right), & \text { if } b \in \mathbf{B}_{u \mid v}^{+}, \\
i_{t_{1}} & \\
\vdots \\
i_{b}
\end{array} \quad \in \mathbf{B}_{m \mid n}\left(\left(1^{t_{b}}\right)\right), \quad \text { if } b \in \mathbf{B}_{u \mid v}^{-} .\right.
$$

So, $\mathbf{i}$ is $\mathfrak{g l}_{m \mid n}$-equivalent to

$$
T_{\bar{u}} \otimes T_{\overline{u-1}} \otimes \cdots \otimes T_{v-1} \otimes T_{v}
$$

where $T_{b}=\emptyset$ if $\mathbf{i}_{b}=\emptyset$. Therefore, if $x_{i}(\mathbf{i}, \mathbf{j}) \neq \mathbf{0}(x=e, f)$ for $i \in I_{m \mid n}$, then $\left(x_{i} \mathbf{i}, \mathbf{j}\right) \in$ $\Omega$. It follows that $\Omega$ is a crystal graph for $\mathfrak{g l}_{m \mid n}$.

\subsection{Bicrystal graphs}

Consider the following set of matrices of non-negative integers;

$$
\begin{aligned}
\mathbf{M}_{m|n, u| v}= & \left\{A=\left(a_{b b^{\prime}}\right)_{b \in \mathbf{B}_{m \mid n}, b^{\prime} \in \mathbf{B}_{u \mid v}} \mid\right. \\
& \text { (1) } \left.a_{b b^{\prime}} \in \mathbb{Z}_{\geq 0} \text {, (2) } a_{b b^{\prime}} \leq 1 \quad \text { if }|b| \neq\left|b^{\prime}\right|\right\} .
\end{aligned}
$$

For simplicity, we write $\mathbf{M}=\mathbf{M}_{m|n, u| v}$.

For $(\mathbf{i}, \mathbf{j}) \in \Omega$, define $A(\mathbf{i}, \mathbf{j})=\left(a_{b b^{\prime}}\right)$ to be the matrix in $\mathbf{M}$, where $a_{b b^{\prime}}$ is the number of $k$ 's such that $\left(i_{k}, j_{k}\right)=\left(b, b^{\prime}\right)$ for $b \in \mathbf{B}_{m \mid n}$ and $b^{\prime} \in \mathbf{B}_{u \mid v}$. Then, it follows that the $\operatorname{map}(\mathbf{i}, \mathbf{j}) \mapsto A(\mathbf{i}, \mathbf{j})$ gives a bijection between $\Omega$ and $\mathbf{M}$, where the pair of empty words $(\emptyset, \emptyset)$ corresponds to zero matrix. Similarly, we have a bijection $(\mathbf{k}, \mathbf{1}) \mapsto A(\mathbf{k}, \mathbf{1})$ from $\Omega^{*}$ to $\mathbf{M}$.

With these identifications, $\mathbf{M}$ becomes a crystal graph for both $\mathfrak{g l}_{m \mid n}$ and $\mathfrak{g l}_{u \mid v}$ by Lemma 3.1.

Example 3.2. Suppose that $m|n=u| v=2 \mid 2$ and

$$
A=\left(\begin{array}{ll|ll}
1 & 0 & 1 & 1 \\
0 & 2 & 0 & 0 \\
\hline 1 & 0 & 0 & 1 \\
0 & 0 & 2 & 0
\end{array}\right) \in \mathbf{M}
$$

Then $A=A(\mathbf{i}, \mathbf{j})=A(\mathbf{k}, \mathbf{1})$ for $(\mathbf{i}, \mathbf{j}) \in \Omega$ and $(\mathbf{k}, \mathbf{1}) \in \Omega^{*}$, where

$$
\begin{array}{ll}
\mathbf{i}=1 \overline{2} \overline{1} \overline{1} 222 \overline{2} 1, & \mathbf{k}=\overline{2} \overline{2} \overline{2} \overline{1} \overline{1} 1122, \\
\mathbf{j}=\overline{2} \overline{2} \overline{1} \overline{1} 11122, & \mathbf{1}=21 \overline{2} \overline{1} \overline{1} \overline{2} 211
\end{array}
$$


Definition 3.3. Let $B$ be a crystal graph for both $\mathfrak{g l}_{m \mid n}$ and $\mathfrak{g l}_{u \mid v}$. We denote by $e_{j}^{*}$ and $f_{j}^{*}$ $\left(j \in I_{u \mid v}\right)$ the Kashiwara operators for $\mathfrak{g l}_{u \mid v}$. We call $B$ a crystal graph for $\mathfrak{g l}_{m \mid n} \oplus \mathfrak{g l}_{u \mid v}$, or $\left(\mathfrak{g l}_{m \mid n}, \mathfrak{g l}_{u \mid v}\right)$-bicrystal if $e_{i}, f_{i}$ commute with $e_{j}^{*}, f_{j}^{*}\left(i \in I_{m \mid n}, j \in I_{u \mid v}\right)$, where we understand the Kashiwara operators as the associated maps from $B \cup\{\boldsymbol{0}\}$ to itself (that is, $x_{i} \mathbf{0}=x_{j}^{*} \mathbf{0}=\mathbf{0}$, for $\left.x=e, f\right)$.

For example, $B=\mathbf{B}_{m \mid n}(\lambda) \times \mathbf{B}_{u \mid v}(\mu)\left(\lambda \in \mathcal{P}_{m \mid n}, \quad \mu \in \mathcal{P}_{u \mid v}\right)$ is a $\left(\mathfrak{g l}_{m \mid n}, \mathfrak{g l}_{u \mid v}\right)$ bicrystal where $x_{i}\left(b_{1}, b_{2}\right)=\left(x_{i} b_{1}, b_{2}\right), x_{j}^{*}\left(b_{1}, b_{2}\right)=\left(b_{1}, x_{j}^{*} b_{2}\right)$ for $\left(b_{1}, b_{2}\right) \in B$ and $x=e, f$.

Lemma 3.4. $\mathbf{M}$ is $a\left(\mathfrak{g l}_{m \mid n}, \mathfrak{g l}_{u \mid v}\right)$-bicrystal.

Proof: The proof is a straightforward verification. So, let us prove the following case;

$$
e_{t}^{*} e_{s} A=e_{s} e_{t}^{*} A
$$

for $A \in \mathbf{M}, 1 \leq s \leq n-1$ and $1 \leq t \leq v-1$. The other cases can be checked in a similar manner.

To show this, we may assume that $A=\left(a_{b b^{\prime}}\right)$ such that $a_{b b^{\prime}}=0$ unless $b \in\{s, s+1\}$ or $b^{\prime} \in\{t, t+1\}$. Put $A^{\prime}=\left(a_{b b^{\prime}}\right)_{b=s, s+1}$ and $A^{\prime \prime}=\left(a_{b b^{\prime}}\right)_{b^{\prime}=t, t+1}$, which are the submatrices of $A$. If neither $A^{\prime}$ nor $A^{\prime \prime}$ contains both an $s$-good - sign and a $t$-good sign of $A$, then (3.4) follows from the fact that the $s$-signature of $e_{t}^{*} A$ is the same as that of $A$, and the $t$-signature of $A$ is the same as that of $e_{s} A$. So we may assume that $A$ is either $A^{\prime}$ or $A^{\prime \prime}$, and prove the case only when $A=A^{\prime}$, that is,

$$
A=\left(\begin{array}{cccccc}
x_{\bar{u}}^{+} & \cdots & x_{\overline{1}}^{+} & x_{1}^{+} & \ldots & x_{v}^{+} \\
x_{\bar{u}}^{-} & \cdots & x_{\overline{1}}^{-} & x_{1}^{-} & \ldots & x_{v}^{-}
\end{array}\right),
$$

where $x_{b}^{+}=a_{s b}$ and $x_{b}^{-}=a_{s+1 b}\left(b \in \mathbf{B}_{u \mid v}\right)$. Note that $A=A(\mathbf{i}, \mathbf{j})=A(\mathbf{k}, \mathbf{1})$ for unique $(\mathbf{i}, \mathbf{j}) \in \Omega$ and $(\mathbf{k}, \mathbf{1}) \in \Omega^{*}$. Then $\mathbf{i}$ and $\epsilon^{(s)}(\mathbf{i})$ are of the form:

$$
\begin{aligned}
\mathbf{i} & =(s+1)^{x_{\bar{u}}^{-}} s^{x_{\bar{u}}^{+}} \ldots(s+1)^{x_{\overline{1}}^{-}} s^{x_{\overline{1}}^{+}} s^{x_{1}^{+}}(s+1)^{x_{1}^{-}} \cdots s^{x_{v}^{+}}(s+1)^{x_{v}^{-}}, \\
\epsilon^{(s)}(\mathbf{i}) & =\left(-{ }^{x_{\bar{u}}^{-}},+^{x_{\bar{u}}^{+}}, \ldots,--^{x_{\overline{1}}^{-}},+^{x_{\overline{1}}^{+}},+^{x_{1}^{+}},--^{x_{1}^{-}}, \ldots,+^{x_{v}^{+}},--^{x_{v}^{-}}\right),
\end{aligned}
$$

where the multiplicities of letters and signs are given as exponents. On the other hand, the $t$-signature of $A$ or $\mathbf{1}$ is completely determined by its subword $\mathbf{1}^{\prime}$ :

$$
\begin{aligned}
\mathbf{1}^{\prime} & =t^{x_{t}^{+}}(t+1)^{x_{t+1}^{+}} t^{x_{t}^{-}}(t+1)^{x_{t+1}^{-}}, \\
\epsilon^{(t)}\left(\mathbf{1}^{\prime}\right) & =\left(+^{x_{t}^{+}},--^{x_{t+1}^{+}},+^{x_{t}^{-}},--^{x_{t+1}^{-}}\right) .
\end{aligned}
$$

For convenience, we set

$$
\tilde{A}=\left(\begin{array}{ll}
a & b \\
c & d
\end{array}\right)=\left(\begin{array}{ll}
x_{t}^{+} & x_{t+1}^{+} \\
x_{t}^{-} & x_{t+1}^{-}
\end{array}\right) .
$$


Case 1. Suppose that $e_{t}^{*} A=\mathbf{0}$ (equivalently, $e_{t}^{*} \tilde{A}=\mathbf{0}$ ). This implies that $d=0$ and $b \leq c$ (note that in this case, we cancel out $(-,+)$ pairs to obtain a $t$-signature). If an $s$-good - sign occurs in $\tilde{A}$, then we have $b<c$ and

$$
e_{s} A=\left(\begin{array}{cccc}
\cdots & a+1 & b & \cdots \\
\cdots & c-1 & 0 & \cdots
\end{array}\right)
$$

Since $b \leq c-1$, we get $e_{t}^{*} e_{s} A=\mathbf{0}$, which implies (3.4). If an $s$-good $-\operatorname{sign}$ of $A$ does not occur in $\tilde{A}$, then $\tilde{A}$ does not change when we apply $e_{s}$ to $A$ and $e_{t}^{*} e_{s} A=$ $\mathbf{0}=e_{s} e_{t}^{*} A$, which also implies (3.4).

Case 2. Suppose that $e_{t}^{*} A \neq \mathbf{0}$ and $b$ is changed by $e_{t}^{*}$. This implies that $b>0$ and $b>c$. If $e_{t}^{*} A=A\left(\mathbf{i}^{\prime}, \mathbf{j}^{\prime}\right)$ for $\left(\mathbf{i}^{\prime}, \mathbf{j}^{\prime}\right) \in \Omega$, then

$$
\begin{aligned}
\epsilon^{(s)}(\mathbf{i}) & =\left(\ldots,+^{a},-^{c},+^{b},-^{d}, \ldots\right), \\
\epsilon^{(s)}\left(\mathbf{i}^{\prime}\right) & =\left(\ldots,+^{a+1},-^{c},+^{b-1},-^{d}, \ldots\right) .
\end{aligned}
$$

Note that the subsequences $\left(+^{a+1},-^{c},+{ }^{b-1},-^{d}\right)$ and $\left(+{ }^{a},-^{c},+^{b},-{ }^{d}\right)$ reduce to the same sequence $\left(+^{a+b-c},-^{d}\right)(a+b-c>0)$, and there is no $s$-good - sign in $c$. If no $s$-good $-\operatorname{sign}$ of $A$ occurs in $\tilde{A}$ (or, in $d$ ), then it is easy to see that (3.4) holds. If there is an $s$-good - sign in $\tilde{A}$, then

$$
\begin{aligned}
& e_{s} e_{t}^{*}\left(\begin{array}{ll}
a & b \\
c & d
\end{array}\right)=e_{s}\left(\begin{array}{cc}
a+1 & b-1 \\
c & d
\end{array}\right)=\left(\begin{array}{cc}
a+1 & b \\
c & d-1
\end{array}\right), \\
& e_{t}^{*} e_{s}\left(\begin{array}{ll}
a & b \\
c & d
\end{array}\right)=e_{t}^{*}\left(\begin{array}{ll}
a & b+1 \\
c & d-1
\end{array}\right)=\left(\begin{array}{cc}
a+1 & b \\
c & d-1
\end{array}\right) .
\end{aligned}
$$

Hence, we have (3.4).

Case 3. Suppose that $e_{t}^{*} A \neq \mathbf{0}$ and $d$ is changed by $e_{t}^{*}$. This implies that $d>0$ and $b \leq c$. If $e_{t}^{*} A=A\left(\mathbf{i}^{\prime}, \mathbf{j}^{\prime}\right)$ for $\left(\mathbf{i}^{\prime}, \mathbf{j}^{\prime}\right) \in \Omega$, then

$$
\begin{aligned}
\epsilon^{(s)}(\mathbf{i}) & =\left(\ldots,+^{a},-^{c},+^{b},-^{d}, \ldots\right), \\
\epsilon^{(s)}\left(\mathbf{i}^{\prime}\right) & =\left(\ldots,+^{a},-^{c+1},+^{b},-{ }^{d-1}, \ldots\right) .
\end{aligned}
$$

Note that the subsequences $\left(+{ }^{a},-{ }^{c+1},+{ }^{b},-{ }^{d-1}\right)$ and $\left(+{ }^{a},-{ }^{c},+^{b},-{ }^{d}\right)$ reduce to the same sequence $\left(+^{a},-^{c-b+d}\right)(c-b+d>0)$. If no $s$-good $-\operatorname{sign}$ of $A$ occurs in $\tilde{A}$ (or, in $d$ ), then it is easy to see that $e_{t}^{*} e_{s} A=e_{s} e_{t}^{*} A$. Assume that an $s$-good sign of $A$ occurs in $\tilde{A}$. If $b=c$, then we have

$$
\begin{aligned}
& e_{s} e_{t}^{*}\left(\begin{array}{ll}
a & b \\
b & d
\end{array}\right)=e_{s}\left(\begin{array}{cc}
a & b \\
b+1 & d-1
\end{array}\right)=\left(\begin{array}{cc}
a+1 & b \\
b & d-1
\end{array}\right), \\
& e_{t}^{*} e_{s}\left(\begin{array}{ll}
a & b \\
b & d
\end{array}\right)=e_{t}^{*}\left(\begin{array}{ll}
a & b+1 \\
b & d-1
\end{array}\right)=\left(\begin{array}{cc}
a+1 & b \\
b & d-1
\end{array}\right) .
\end{aligned}
$$


If $b<c$, then we have

$$
\begin{aligned}
& e_{s} e_{t}^{*}\left(\begin{array}{ll}
a & b \\
c & d
\end{array}\right)=e_{s}\left(\begin{array}{cc}
a & b \\
c+1 & d-1
\end{array}\right)=\left(\begin{array}{cc}
a+1 & b \\
c & d-1
\end{array}\right), \\
& e_{t}^{*} e_{s}\left(\begin{array}{ll}
a & b \\
c & d
\end{array}\right)=e_{t}^{*}\left(\begin{array}{ll}
a+1 & b \\
c-1 & d
\end{array}\right)=\left(\begin{array}{cc}
a+1 & b \\
c & d-1
\end{array}\right) .
\end{aligned}
$$

Hence, we have (3.4).

\section{Lemma 3.5.}

(1) Let $C$ be a connected component in $\mathbf{M}$ as a $\mathfrak{g l}_{m \mid n}$-crystal. If $x_{j}^{*} C \neq\{\mathbf{0}\}$ for some $j \in I_{u \mid v}$ and $x=e, f$, then $x_{j}^{*}: C \rightarrow x_{j}^{*} C$ is an isomorphism of $\mathfrak{g l}_{m \mid n}$-crystals.

(2) Let $C^{*}$ be a connected component in $\mathbf{M}$ as a $\mathfrak{g l}_{u \mid v}$-crystal. If $x_{i} C^{*} \neq\{\mathbf{0}\}$ for some $i \in I_{m \mid n}$ and $x=e, f$, then $x_{i}: C^{*} \rightarrow x_{i} C^{*}$ is an isomorphism of $\mathfrak{g l}_{u \mid v}$-crystals.

Proof: (1) Choose $b \in C$ such that $x_{j}^{*} b \neq \mathbf{0}$. For any $b^{\prime} \in C$, we have

$$
b^{\prime}=x_{i_{1}} \ldots x_{i_{t}} b
$$

for some Kashiwara operators $x_{i_{k}}\left(i_{k} \in I_{m \mid n}, 1 \leq k \leq t\right)$. We first claim that $x_{j}^{*} b^{\prime} \neq \mathbf{0}$.

We will use induction on $t$. Suppose that $t=1$. Let $y_{i_{1}}$ be the Kashiwara operator given by $y_{i_{1}}=f_{i_{1}}\left(\right.$ resp. $\left.y_{i_{1}}=e_{i_{1}}\right)$ if $x_{i_{1}}=e_{i_{1}}\left(\right.$ resp. $\left.x_{i_{1}}=f_{i_{1}}\right)$. Then $b=y_{i_{1}} b^{\prime}$, and

$$
\mathbf{0} \neq x_{j}^{*} b=x_{j}^{*} y_{i_{1}} b^{\prime}=y_{i_{1}} x_{j}^{*} b^{\prime}
$$

by Lemma 3.4, which implies that $x_{j}^{*} b^{\prime} \neq \mathbf{0}$. Suppose that $t>1$. Since $x_{j}^{*} x_{i_{t}} b \neq \mathbf{0}$ and $b^{\prime}=x_{i_{1}} \ldots x_{i_{t-1}}\left(x_{i_{t}} b\right)$, it follows that $x_{j}^{*} b^{\prime} \neq \mathbf{0}$ by induction hypothesis. This completes the induction.

Hence, the composite of the following two maps is the identity map on $C$;

$$
C \stackrel{x_{j}^{*}}{\longrightarrow} x_{j}^{*} C \stackrel{y_{j}^{*}}{\longrightarrow} C
$$

where $y_{j}^{*}=e_{j}^{*}\left(\operatorname{resp} . f_{j}^{*}\right)$ if $x_{j}^{*}=f_{j}^{*}\left(\right.$ resp. $e_{j}^{*}$ ). This implies that $x_{j}^{*}$ is a bijection which commutes with $e_{i}, f_{i}\left(i \in I_{m \mid n}\right)$ by Lemma 3.4, and that $x_{j}^{*} C$ is isomorphic to $C$ as a $\mathfrak{g l}_{m \mid n}$-crystal. The proof of (2) is similar.

Given $A \in \mathbf{M}$, suppose that $A=A(\mathbf{i}, \mathbf{j})=A(\mathbf{k}, \mathbf{1})$ for $(\mathbf{i}, \mathbf{j}) \in \Omega$ and $(\mathbf{k}, \mathbf{1}) \in \Omega^{*}$. We define

$$
\pi(A)=\left(P_{1}(A), P_{2}(A)\right)=(P(\mathbf{i}), P(\mathbf{1}))
$$

(see (2.1)). Then $\pi(A) \in \mathbf{B}_{m \mid n}(\lambda) \times \mathbf{B}_{u \mid v}(\mu)$ for some $(\lambda, \mu) \in \mathcal{P}_{m \mid n} \times \mathcal{P}_{u \mid v}$. Note that $A \simeq_{\mathfrak{g l}_{m \mid n}} P_{1}(A)$, and $A \simeq_{\mathfrak{g l}_{u \mid v}} P_{2}(A)$. 
Lemma 3.6. Suppose that $A \in \mathbf{M}$ is given.

(1) If $x_{j}^{*} A \neq \mathbf{0}$ for some $j \in I_{u \mid v}$ and $x=e, f$, then $P_{1}\left(x_{j}^{*} A\right)=P_{1}(A)$.

(2) If $x_{i} A \neq \mathbf{0}$ for some $i \in I_{m \mid n}$ and $x=e, f$, then $P_{2}\left(x_{i} A\right)=P_{2}(A)$.

Proof: It suffices to prove (1). By Lemma 3.5, $A$ is $\mathfrak{g l}_{m \mid n}$-equivalent to $x_{j}^{*} A$, and hence the tableaux $P_{1}(A)$ and $P_{1}\left(x_{j}^{*} A\right)$ are $\mathfrak{g l}_{m \mid n}$-equivalent. By Lemma 2.9, we have $P_{1}\left(x_{j}^{*} A\right)=P_{1}(A)$.

Let $B$ be a $\left(\mathfrak{g l}_{m \mid n}, \mathfrak{g l}_{u \mid v}\right)$-bicrystal. If we set $\mathcal{I}=I_{m \mid n} \sqcup I_{u \mid v}^{*}$ where $I_{u \mid v}^{*}=\left\{j^{*} \mid j \in\right.$ $\left.I_{u \mid v}\right\}$, then $B$ is an $\mathcal{I}$-colored oriented graph with respect to $x_{i}, x_{j}^{*}$ for $i \in I_{m \mid n}, j \in I_{u \mid v}$, and $x=e, f$. Now, we can characterize a connected component in $\mathbf{M}$ as a $\left(\mathfrak{g l}_{m \mid n}, \mathfrak{g l}_{u \mid v}\right)$ bicrystal.

Proposition 3.7. For each connected component $\mathbf{C}$ in $\mathbf{M}$ as a $\left(\mathfrak{g l}_{m \mid n}, \mathfrak{g l}_{u \mid v}\right)$-bicrystal, $\pi$ gives the following isomorphism of $\left(\mathfrak{g l}_{m \mid n}, \mathfrak{g l}_{u \mid v}\right)$-bicrystals;

$$
\pi: \mathbf{C} \longrightarrow \mathbf{B}_{m \mid n}(\lambda) \times \mathbf{B}_{u \mid v}(\mu),
$$

for some $\lambda \in \mathcal{P}_{m \mid n}$ and $\mu \in \mathcal{P}_{u \mid v}$.

Proof: By Lemma 3.6, we have

$$
\pi\left(x_{i} A\right)=\left(x_{i} P_{1}(A), P_{2}(A)\right)
$$

for $A \in \mathbf{M}, i \in I_{m \mid n}$ and $x=e, f$ (we assume that $\pi(\mathbf{0})=\mathbf{0}$ and $\left.\left(\mathbf{0}, P_{2}(A)\right)=\mathbf{0}\right)$. Similarly, we have $\pi\left(x_{j}^{*} A\right)=\left(P_{1}(A), x_{j} P_{2}(A)\right)$ for $j \in I_{u \mid v}$.

Let $\mathbf{C}$ be a connected component in $\mathbf{M}$ as an $\mathcal{I}$-colored oriented graph. Choose an arbitrary $A \in \mathbf{C}$. Suppose that $\pi(A) \in \mathbf{B}_{m \mid n}(\lambda) \times \mathbf{B}_{u \mid v}(\mu)$ for some $\lambda \in \mathcal{P}_{m \mid n}$ and $\mu \in \mathcal{P}_{u \mid v}$. Then we have

$$
\pi: \mathbf{C} \longrightarrow \mathbf{B}_{m \mid n}(\lambda) \times \mathbf{B}_{u \mid v}(\mu),
$$

where $\pi$ commutes with $e_{i}, f_{i}$ and $e_{j}^{*}, f_{j}^{*}\left(i \in I_{m \mid n}, j \in I_{u \mid v}\right)$. It is clear that $\pi$ is onto.

Now suppose that $\pi(A)=\pi\left(A^{\prime}\right)$ for some $A, A^{\prime} \in \mathbf{C}$. Since $\mathbf{C}$ is connected,

$$
x_{j_{1}}^{*} \ldots x_{j_{q}}^{*} x_{i_{1}} \ldots x_{i_{p}} A=A^{\prime}
$$

for some Kashiwara operators $x_{i_{k}}\left(i_{k} \in I_{m \mid n}, 1 \leq k \leq p\right), x_{j_{l}}^{*}\left(j_{l} \in I_{u \mid v}, 1 \leq l \leq q\right)$. Put $A^{\prime \prime}=x_{i_{1}} \cdots x_{i_{p}} A$. Then $A$ and $A^{\prime \prime}$ belong to the same connected component as a $\mathfrak{g l}_{m \mid n}$-crystal, say $C_{1}$. On the other hand, by Lemma 3.6, we have

$$
P_{1}\left(A^{\prime \prime}\right)=P_{1}\left(A^{\prime}\right)=P_{1}(A)
$$

Since the map $P_{1}: C_{1} \rightarrow \mathbf{B}_{m \mid n}(\lambda)$ is an isomorphism of $\mathfrak{g l}_{m \mid n}$-crystals, it follows that $A^{\prime \prime}=A$. 
Next, $A^{\prime \prime}$ and $A^{\prime}$ belong to the same connected component as a $\mathfrak{g l}_{u \mid v}$-crystal, say $C_{2}$. By Lemma 3.6, we have

$$
P_{2}\left(A^{\prime \prime}\right)=P_{2}(A)=P_{2}\left(A^{\prime}\right) .
$$

Since the map $P_{2}: C_{2} \rightarrow \mathbf{B}_{u \mid v}(\mu)$ is an isomorphism, we have $A^{\prime \prime}=A^{\prime}$, and hence $A=A^{\prime}$. So, $\pi$ is one-to-one.

Therefore, $\pi$ is an isomorphism of $\left(\mathfrak{g l}_{m \mid n}, \mathfrak{g l}_{u \mid v}\right)$-bicrystals.

Example 3.8. Let $A$ be as in Example 3.2. Then

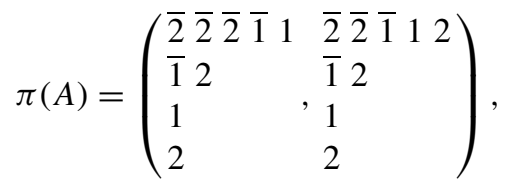

and the connected component of $A$ is isomorphic to $\mathbf{B}_{2 \mid 2}(5,2,1,1) \times \mathbf{B}_{2 \mid 2}(5,2,1,1)$.

\subsection{Decomposition of $\mathbf{M}$}

Now, we will describe an explicit decomposition of M. Set

$$
\mathbf{M}_{\text {h.w. }}=\left\{A \in \mathbf{M} \mid \pi(A)=\left(H_{m \mid n}^{\lambda}, H_{u \mid v}^{\mu}\right) \text { for some }(\lambda, \mu) \in \mathcal{P}_{m \mid n} \times \mathcal{P}_{u \mid v}\right\},
$$

which is the set of all the highest weight elements in $\mathbf{M}$. We have seen in the proof of Proposition 3.7 that $\pi$ induces an isomorphism of $\left(\mathfrak{g l}_{m \mid n}, \mathfrak{g l}_{u \mid v}\right)$-bicrystals between the connected components of $A \in \mathbf{M}$ and $\pi(A)$. Hence,

$$
\mathbf{M}=\bigoplus_{A \in \mathbf{M}_{\text {h.w. }}} \mathbf{C}(A)
$$

where $\mathbf{C}(A)$ is the connected component of $A$ in $\mathbf{M}$, which is isomorphic to $\mathbf{B}_{m \mid n}(\lambda) \times$ $\mathbf{B}_{u \mid v}(\mu)$ for some $\lambda \in \mathcal{P}_{m \mid n}$ and $\mu \in \mathcal{P}_{u \mid v}$.

Suppose that $\lambda=\left(\lambda_{1}, \ldots, \lambda_{r}\right) \in \mathcal{P}_{m \mid n} \cap \mathcal{P}_{u \mid v}$ is given. Let $v=\left(v_{1}, v_{2}, \ldots, v_{\ell}\right)$ be the sequence of non-negative integers $\left(\ell=\lambda_{m+1}\right)$ determined by

$$
\left(v_{1}+\cdots+v_{\ell}, v_{2}+\cdots+v_{\ell}, \ldots, v_{\ell}\right)=\left(\lambda_{m+1}, \ldots, \lambda_{r}\right)^{\prime}
$$

where $\left(\lambda_{m+1}, \ldots, \lambda_{r}\right)^{\prime}$ is the conjugate of the partition $\left(\lambda_{m+1}, \ldots, \lambda_{r}\right)$ (cf. [18]).

Assume that $m \geq u$. Let us define $A_{\lambda}=\left(a_{b b^{\prime}}\right) \in \mathbf{M}$ by

(1) for $0 \leq k<m, 0 \leq l<u$,

$$
a_{\overline{m-k}} \overline{u-l}= \begin{cases}\lambda_{k+1}, & \text { if } 0 \leq k=l<u, \\ 0, & \text { otherwise }\end{cases}
$$


(2) for $0 \leq k<m, 1 \leq t \leq v$,

$$
a_{\overline{m-k} t}= \begin{cases}1, & \text { if } u \leq k<m \text { and } \lambda_{k+1} \geq t \\ 0, & \text { otherwise }\end{cases}
$$

(3) for $1 \leq s \leq n, 0 \leq l<u$, $a_{s \overline{u-l}}=0$.

(4) for $1 \leq s \leq n, 1 \leq t \leq v$,

$$
a_{s t}= \begin{cases}v_{s+t-1}, & \text { if } 2 \leq s+t \leq \ell+1 \\ 0, & \text { otherwise }\end{cases}
$$

Note that $A_{\lambda}$ is of the following form;

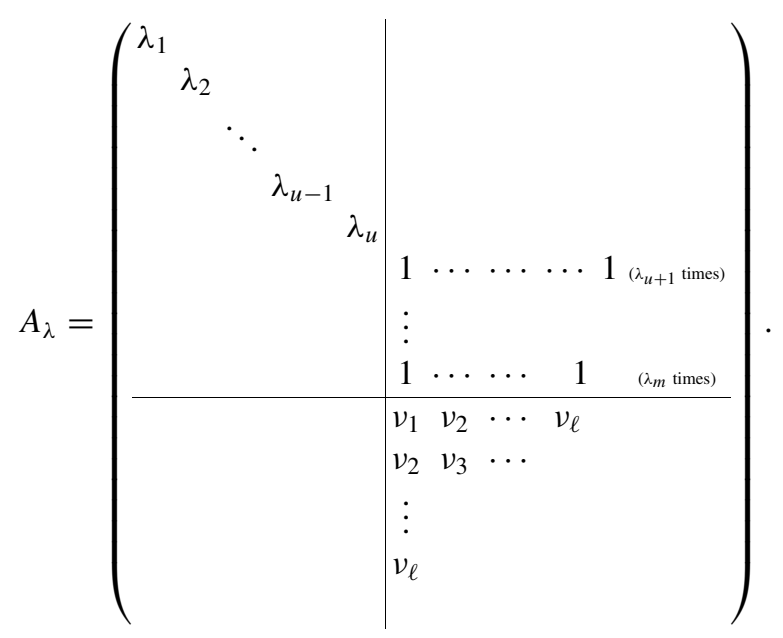

If $m \leq u$, then we define $A_{\lambda}$ to be the transpose of (3.8), where $m$ and $u$ are exchanged. Note that $A_{\lambda}$ is uniquely determined by $\lambda$. By Schensted's column bumping algorithm, it is not difficult to see that

Lemma 3.9. For $\lambda \in \mathcal{P}_{m \mid n} \cap \mathcal{P}_{u \mid v}$, we have

$$
\pi\left(A_{\lambda}\right)=\left(H_{m \mid n}^{\lambda}, H_{u \mid v}^{\lambda}\right) \in \mathbf{B}_{m \mid n}(\lambda) \times \mathbf{B}_{u \mid v}(\lambda)
$$


Example 3.10. Suppose that $m|n=4| 3$ and $u|v=3| 3$. Let $\lambda=(7,5,5,3,3,2,2,1)$. Then

$$
A_{\lambda}=\left(\begin{array}{lll|lll}
7 & 0 & 0 & 0 & 0 & 0 \\
0 & 5 & 0 & 0 & 0 & 0 \\
0 & 0 & 5 & 0 & 0 & 0 \\
0 & 0 & 0 & 1 & 1 & 1 \\
\hline 0 & 0 & 0 & 1 & 2 & 1 \\
0 & 0 & 0 & 2 & 1 & 0 \\
0 & 0 & 0 & 1 & 0 & 0
\end{array}\right),
$$

and

$$
\pi\left(A_{\lambda}\right)=\left(\begin{array}{llllllllllllll}
\overline{4} & \overline{4} & \overline{4} & \overline{4} & \overline{4} & \overline{4} & \overline{4} & \overline{3} & \overline{3} & \overline{3} & \overline{3} & \overline{3} & \overline{3} & \overline{3} \\
\overline{3} & \overline{3} & \overline{3} & \overline{3} & \overline{3} & & \overline{2} & \overline{2} & \overline{2} & \overline{2} & \overline{2} & & \\
\overline{2} & \overline{2} & \overline{2} & \overline{2} & \overline{2} & & \overline{1} & \overline{1} & \overline{1} & \overline{1} & \overline{1} & \\
\overline{1} & \overline{1} & \overline{1} & & & & 1 & 2 & 3 & & & \\
1 & 2 & 3 & & & & 1 & 2 & 3 & & & \\
1 & 2 & & & & & 1 & 2 & & & & \\
1 & 2 & & & & 1 & 2 & & & & \\
1 & & & & & 1 & & & & &
\end{array}\right)
$$

Now, we can state one of our main results, which characterizes all the highest weight elements in $\mathbf{M}$. This is a natural super analogue of the result in [6], which describes the decomposition of $\mathbf{M}_{m|0, u| 0}$ as a $\left(\mathfrak{g l}_{m}, \mathfrak{g l}_{u}\right.$ )-bicrystal (that is, $\mathfrak{g l}_{m}=\mathfrak{g l}_{m \mid 0}$ and $\left.\mathfrak{g l}_{u}=\mathfrak{g l}_{u \mid 0}\right)$. In case of $\mathbf{M}_{m|0, u| 0}$, the set of highest weight vectors can be found directly since a matrix $A$ corresponds to a highest weight element if and only if $e_{i} A=e_{k}^{*} A=\mathbf{0}$ for all $i \in I_{m \mid 0}$ and $k \in I_{u \mid 0}$. But in super case, this is no longer true because of fake highest weight vectors (see Remark 2.7). So, we need more additional arguments to exclude the elements in $\mathbf{M}$ corresponding to fake highest weight vectors.

Theorem 3.11 (cf. [6]). We have

$$
\mathbf{M}_{\text {h.w. }}=\left\{A_{\lambda} \mid \lambda \in \mathcal{P}_{m \mid n} \cap \mathcal{P}_{u \mid v}\right\},
$$

and hence the following isomorphism of $\left(\mathfrak{g l}_{m \mid n}, \mathfrak{g l}_{u \mid v}\right)$-bicrystals;

$$
\pi: \mathbf{M} \longrightarrow \bigoplus_{\lambda \in \mathcal{P}_{m \mid n} \cap \mathcal{P}_{u \mid v}} \mathbf{B}_{m \mid n}(\lambda) \times \mathbf{B}_{u \mid v}(\lambda)
$$

Proof: For convenience, we assume that $m \geq u$. Suppose that $A=\left(a_{b b^{\prime}}\right) \in \mathbf{M}_{\text {h.w. }}$ is given. Suppose that $A=A(\mathbf{i}, \mathbf{j})=A(\mathbf{k}, \mathbf{1})$ for $(\mathbf{i}, \mathbf{j}) \in \Omega,(\mathbf{k}, \mathbf{1}) \in \Omega^{*}$, and $\mathbf{i}=i_{1} \ldots i_{r}$ and $\mathbf{j}=j_{1} \ldots j_{r}$ for some $r \geq 1$. We claim that $A=A_{\lambda}$ for some $\lambda \in \mathcal{P}_{m \mid n} \cap \mathcal{P}_{u \mid v}$ (see (3.8)). We may assume that $A$ is a non-zero matrix since the zero matrix corresponds to $A_{(0)}$. Recall that $P(\mathbf{i})$ (resp. $P(\mathbf{1})$ ) is a highest weight tableau, which implies that Springer 
$e_{i} A=e_{j}^{*} A=\mathbf{0}$ for all $i \in I_{m \mid n}$ and $j \in I_{u \mid v}$. Also, the following fact is of important use in the proof;

(H) no element $b \in \mathbf{B}_{m \mid n}^{-}$of $\mathbf{i}$ (resp. $b \in \mathbf{B}_{u \mid v}^{-}$of $\mathbf{1}$ ) is bumped into the first $m$ rows (resp. $u$ rows) at each step of insertions when we get $P(\mathbf{i})($ resp. $P(\mathbf{1})$ ).

We will divide $A$ into several submatrices and prove that each submatrix is of the form given in (3.11).

Step 1. In this step, we will show that (i) $a_{b b^{\prime}}=0$ if either $b \in\{\bar{m}, \ldots, \overline{m-u+1}\}$, or $b^{\prime} \in\{\bar{u}, \ldots, \overline{1}\}$ except the diagonal entries, and (ii) $a_{\bar{m} \bar{u}} \geq \cdots \geq a_{\overline{m-u+1}}$. Let us write

$$
\mathbf{i}=\mathbf{i}_{u} \mathbf{i}_{u-1} \cdots \mathbf{i}_{v-1} \mathbf{i}_{v},
$$

where $\mathbf{i}_{b}=i_{t_{1}} \ldots i_{t_{b}}\left(b \in \mathbf{B}_{u \mid v}, t_{b} \geq 0\right)$ is a subword of $\mathbf{i}$ such that $j_{t_{1}}=\cdots=j_{t_{b}}=$ $b$. Similarly, we write $\mathbf{1}=\mathbf{1}_{\bar{m}} \cdots \mathbf{1}_{n}$.

Since $A \in \mathbf{M}_{\text {h.w. }}, \pi(A)=(P(\mathbf{i}), P(\mathbf{1}))$ is a pair of highest weight tableaux. By Schensted's algorithm, we observe that the shape of $P\left(\mathbf{i}_{\bar{u}}\right)$ is a single row, and all the letters in $\mathbf{i}_{\bar{u}}$ are placed in the first row of $P(\mathbf{i})$. Hence, we should have $\mathbf{i}_{\bar{u}}=\bar{m} \cdots \bar{m}$ ( $a_{\bar{m} \bar{u}}$ times), equivalently, $a_{b \bar{u}}=0$ for all $b \in \mathbf{B}_{m \mid n} \backslash\{\bar{m}\}$ (see Fig. 1). By the same argument, we have $\mathbf{1}_{\bar{m}}=\bar{u} \cdots \bar{u}\left(a_{\bar{m} \bar{u}}\right.$ times), or $a_{\bar{m} b}=0$ for all $b \in \mathbf{B}_{u \mid v} \backslash\{\bar{u}\}$.

Next, consider $\mathbf{i}_{\overline{u-1}}$. Since $a_{\bar{m} \overline{u-1}}=0$, all the letters in $\mathbf{i}_{\overline{u-1}}$ are greater than $\bar{m}$, and placed in the first two rows of $P(\mathbf{i})$. This implies that $\mathbf{i}_{\overline{u-1}}=$ $\overline{m-1} \cdots \overline{m-1}\left(a_{\overline{m-1}} \overline{u-1}\right.$ times), and $a_{\bar{m} \bar{u}} \geq a_{\overline{m-1}} \overline{u-1}$ (see Fig. 1). Similarly, $\mathbf{1}_{\overline{m-1}}=\overline{u-1} \cdots \overline{u-1}\left(a_{\overline{m-1}} \overline{u-1}\right.$ times). Repeating the above argument, it follows that for $0 \leq k \leq u-1$,

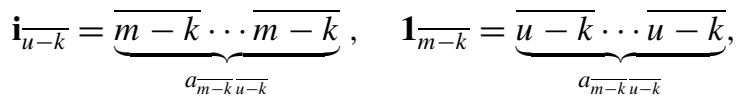

and

$$
\lambda_{1} \geq \cdots \geq \lambda_{u}
$$

where $\lambda_{k+1}=a_{\overline{m-k}} \overline{u-k}$ for $0 \leq k \leq u-1$.

Step 2. Suppose that $a_{s t} \neq 0$ for some $1 \leq s \leq n$ and $1 \leq t \leq v$. Let $\ell$ be the maximum column index $(1 \leq \ell \leq v)$ such that $a_{s \ell} \neq 0$ for some $1 \leq s \leq n$. In this step, we will show that (i) $a_{s t}=0$ for $s+t>\ell+1$, and (ii) $a_{s t}=a_{s^{\prime} t^{\prime}}$ for $2 \leq s+t=$ $s^{\prime}+t^{\prime} \leq \ell+1$.

First, we claim that $a_{s t}=0$ for $s+t>\ell+1$. Consider $a_{s \ell}$ for $1 \leq s \leq n$. Suppose that $a_{s \ell} \neq 0$ for some $s \geq 2$. Then we have $e_{s-1} A \neq \mathbf{0}$, since $\epsilon^{(s-1)}(A)=$ $\left(\ldots,+{ }^{a_{s-1 \ell}},-{ }^{a_{s \ell}}\right)$ and there exists at least one - in the $(s-1)$-signature of $A$. This is a contradiction. So, we have $a_{1 \ell} \neq 0$ and $a_{s \ell}=0$ for $s \geq 2$. Next, consider $a_{s \ell-1}$ for $1 \leq s \leq n$. Suppose that $a_{s \ell-1} \neq 0$ for some $s \geq 3$. Then we also have $e_{s-1} A \neq \mathbf{0}$, since $\epsilon^{(s-1)}(A)=\left(\ldots,+^{a_{s-1 \ell-1}},-^{a_{s \ell-1}}\right)$, which is a contradiction. Similarly, we can check that $a_{s t}=0$ for $1+\min \{\ell-n, 0\} \leq t \leq \ell$ and $s>\ell-t+1$.

Now, we claim that $a_{s t}=a_{s^{\prime} t^{\prime}}$ for $2 \leq s+t=s^{\prime}+t^{\prime} \leq \bar{\ell}+1$. 
Consider $a_{s t}$ for $s+t=\ell+1$. Since $e_{1} A=\mathbf{0}$ and $e_{\ell-1}^{*} A=\mathbf{0}$, we have $a_{2 \ell-1} \leq$ $a_{1 \ell}$ and $a_{2 \ell-1} \geq a_{1 \ell}$, respectively. Hence $a_{2 \ell-1}=a_{1 \ell}$. Continuing this argument, it follows that $a_{s \ell-s+1}=a_{1 \ell}$ for $1 \leq s \leq \min \{\ell, n\}$. Indeed, we have $\ell \leq n$. Otherwise, we have $a_{n \ell-n+1} \neq 0$, and $e_{\ell-n}^{*} A \neq \mathbf{0}$. Next, consider $a_{s t}$ for $s+t=\ell$. Since $e_{1} A=\mathbf{0}$ and

$$
\epsilon^{(1)}(A)=\left(\ldots,-{ }^{a_{2 \ell-2}},+{ }^{a_{1 \ell-1}},-{ }^{a_{2 \ell-1}},+{ }^{a_{1 \ell}}\right)
$$

(note that $a_{2 \ell-1}=a_{1 \ell}$ ), we have $a_{2 \ell-2} \leq a_{1 \ell-1}$. Since $e_{\ell-2}^{*} A=\mathbf{0}$ and

$$
\epsilon^{(\ell-2)}(A)=\left(\ldots,-{ }^{a_{1 \ell-1}},+^{a_{2 \ell-2}},-^{a_{2 \ell-1}},+^{a_{3 \ell-2}}\right)
$$

(note that $a_{3 \ell-2}=a_{2 \ell-1}=a_{1 \ell}$ ), we have $a_{2 \ell-2} \geq a_{1 \ell-1}$. Hence, $a_{2 \ell-2}=a_{1 \ell-1}$. Similarly, we can check that $a_{1 \ell-1}=a_{2 \ell-2}=a_{3 \ell-3}=\cdots=a_{\ell-11}$.

Applying the above argument successively, we conclude that $a_{s t}=a_{s^{\prime} t^{\prime}}$ for $2 \leq$ $s+t=s^{\prime}+t^{\prime} \leq \ell+1$. We set $v_{k}=a_{1 k}$ for $1 \leq k \leq \ell$.

Step 3. Let $\ell$ be the maximum column index $(1 \leq \ell \leq v)$ given in Step 2. We assume that $\ell=0$ if $a_{s t}=0$ for all $1 \leq s \leq n$ and $1 \leq t \leq v$. We claim that $a_{\bar{k} t}=1$ for $1 \leq k \leq m-u$ and $1 \leq t \leq \ell$.

Let us use the induction on $t$. If $\ell=0$, then it is clear. Suppose that $\ell>0$. Consider $P_{1}=P\left(\mathbf{i}_{\bar{u}} \ldots \mathbf{i}_{\overline{1}} \mathbf{i}_{1}\right)$. We have seen in Step 1 that $P\left(\mathbf{i}_{\bar{u}} \ldots \mathbf{i}_{\overline{1}}\right)$ is an $(m, n)$ hook semistandard tableau whose shape is a partition $\left(\lambda_{1}, \ldots, \lambda_{u}\right)$ with the $k^{\text {th }}$ row filled with $\overline{m-k+1}(1 \leq k \leq u)$. When we insert the word $\mathbf{i}_{1}$ into $P\left(\mathbf{i}_{\bar{u}} \ldots \mathbf{i}_{\overline{1}}\right)$, all the letters in $\mathbf{i}_{1}$ are placed in the first column of $P_{1}$. If $a_{\bar{k} 1}=0$ for some $1 \leq k \leq$ $m-u$, then there exists at least one letter in $\mathbf{B}_{m \mid n}^{-}$placed in the first $m$ rows of $P_{1}$ and hence $P(\mathbf{i})$. This contradicts the fact that $P(\mathbf{i})$ is a highest weight tableau (recall (H)).

For $t<\ell$, suppose that $a_{\bar{k} t^{\prime}}=1$ for $1 \leq k \leq m-u$ and $1 \leq t^{\prime} \leq t$. Put $P_{t}=$ $P\left(\mathbf{i}_{\bar{u}} \ldots \mathbf{i}_{\overline{1}} \mathbf{i}_{1} \ldots \mathbf{i}_{t}\right)$. Then, each $k^{\text {th }}$ row of $P_{t}(1 \leq k \leq m)$ is filled with $\overline{m-k+1}$. If we cut out the first $m$ rows of $P_{t}$, then the remaining tableau consists of exactly $t$ columns. Moreover, if we read its $k^{\text {th }}$ column $(1 \leq k \leq t)$ from top to bottom, then the associated word is given by

$$
k^{v_{k}+\cdots+v_{t}}(k+1)^{v_{t+1}}(k+2)^{v_{t+2}} \cdots(\ell-t+k)^{v_{\ell}} .
$$

Since

$$
\mathbf{i}_{t+1}=(\overline{m-u})^{a_{\overline{m-u} t+1}} \cdots \overline{1}^{a_{\overline{1} t+1}} 1^{v_{t+1}} \cdots(\ell-t)^{\nu_{\ell}},
$$

it is not difficult to see that for $1 \leq k \leq \ell-t, v_{t+k}(t+k)$ 's are bumped out of the $t^{\text {th }}$ column and placed in the $(t+1)^{\text {st }}$ column, when we insert the word $\mathbf{i}_{t+1}$ into $P_{t}$ (note that $v_{\ell}>0$ and $\mathbf{i}_{t+1}$ is not an empty word). So, if $a_{\bar{k} t+1}=0$ for some $1 \leq k \leq m-u$, then at least one letter in $\mathbf{B}_{m \mid n}^{-}$happens to be placed in the first $m$ rows of $P_{t+1}=P\left(\mathbf{i}_{\bar{u}} \ldots \mathbf{i}_{\overline{1}} \mathbf{i}_{1} \ldots \mathbf{i}_{t+1}\right)$ and hence $P(\mathbf{i})$, which is a contradiction. This completes the induction. 
Step 4. Finally, we will show that (i) if $a_{\overline{1} t}=0$ for some $\ell<t<v$, then $a_{\overline{1} t+1}=0$., and (ii) if $a_{\bar{k} t}=0$ for some $1<k \leq m-u$ and $\ell<t<v$, then $a_{\bar{k} t+1}=a_{\overline{k-1} t}=0$.

Consider $a_{\overline{1} t}$ for $\ell<t \leq v$. Suppose that $a_{\overline{1} t}=0$ and $a_{\overline{1} t+1}=1$ for some $\ell<$ $t<v$. Then, we have $e_{t}^{*} A \neq \mathbf{0}$ since $\epsilon^{(t)}(A)=(\ldots,-)$ has a $t$-good - sign, which is a contradiction.

Next, consider $a_{\overline{2} t}$ for $\ell<t \leq v$. Suppose that $a_{\overline{2}_{t}}=0$ and $a_{\overline{2}_{t+1}}=1$ for some $\ell<t<v$. If $a_{\overline{1} t}=a_{\overline{1} t+1}=0$, then $e_{t}^{*} A \neq \mathbf{0}$. Otherwise, we can check that there exists a $\overline{1}$-good - sign in $A$ with respect to the $\mathfrak{g l}_{m \mid n}$-crystal structure, and $e_{\overline{1}} A \neq \mathbf{0}$. So, in any case, we get a contradiction. Then, in a similar way, we see that there is no $t(\ell<t \leq v)$ such that $a_{\overline{2} t}=0$ and $a_{\overline{1} t}=1$, since $e_{\overline{1}} A=0$.

Now, applying the above arguments successively to $a_{\bar{k} t}$ for $1<k \leq m-u$ and $\ell<t \leq v$, it follows that if $a_{\bar{k} t}=0$ for $1<k \leq m-u$ and $\ell<t<v$, then $a_{\bar{k} t+1}=$ $a_{\overline{k-1} t}=0$. This leads to

$$
\lambda_{u+1} \geq \cdots \geq \lambda_{m}
$$

where $\lambda_{u+k}=\sum_{1 \leq t \leq v} a_{\overline{m-u-k+1} t}(1 \leq k \leq m-u)$. Also, we have $\lambda_{u} \geq \lambda_{u+1}$ since $e_{\overline{m-u}} A=0$.

Let $\left(\lambda_{m+1}, \lambda_{m+2}, \ldots, \lambda_{r}\right)$ be the partition determined by (3.7), and put

$$
\lambda=\left(\lambda_{1}, \ldots, \lambda_{m}, \lambda_{m+1}, \ldots, \lambda_{r}\right) .
$$

Note that $\lambda_{m+1}=\ell$, where $\ell$ is the maximum column index given in Step 2, and $\lambda_{m} \geq$ $\lambda_{m+1}$ by Step 3 . So, $\lambda$ is a Young diagram, and $\lambda \in \mathcal{P}_{m \mid n} \cap \mathcal{P}_{u \mid v}$ by construction. Finally, we conclude that $A=A_{\lambda}$ given in (3.8).

Let $x=\left\{x_{b} \mid b \in \mathbf{B}_{m \mid n}\right\}$ and $y=\left\{y_{b^{\prime}} \mid b^{\prime} \in \mathbf{B}_{u \mid v}\right\}$. The character of $\mathbf{M}$ is given by

$$
\operatorname{ch} \mathbf{M}=\sum_{A \in \mathbf{M}} x^{\mathrm{wt}(A)} y^{\mathrm{wt}^{*}(A)}=\frac{\prod_{|b| \neq\left|b^{\prime}\right|}\left(1+x_{b} y_{b^{\prime}}\right)}{\prod_{|b|=\left|b^{\prime}\right|}\left(1-x_{b} y_{b^{\prime}}\right)},
$$

where $b \in \mathbf{B}_{m \mid n}$ and $b^{\prime} \in \mathbf{B}_{u \mid v}$. By Theorem 3.11, we recover the super Cauchy identity;

$$
\frac{\prod_{|b| \neq\left|b^{\prime}\right|}\left(1+x_{b} y_{b^{\prime}}\right)}{\prod_{|b|=\left|b^{\prime}\right|}\left(1-x_{b} y_{b^{\prime}}\right)}=\sum_{\lambda \in \mathcal{P}_{m \mid n} \cap \mathcal{P}_{u \mid v}} h s_{\lambda}(x) h s_{\lambda}(y) .
$$

Remark 3.12. Let $\mathcal{A}_{n}=\left\{a_{1}<\cdots<a_{n}\right\}$ be the set of $n$ letters with a linear ordering. For a partition $\lambda$ with length at most $n$, a tableau $T$ obtained by filling $\lambda$ with the letters in $\mathcal{A}_{n}$ is called a semistandard tableau of shape $\lambda$ if the entries in each row (resp. column) are weakly (resp. strictly) increasing from left to right (resp. from top

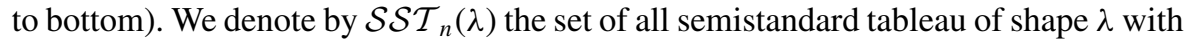
entries in $\mathcal{A}_{n}$. For example, $\mathbf{B}_{m \mid 0}(\lambda)\left(\lambda \in \mathcal{P}_{m \mid 0}\right)$ may be identified with $\mathcal{S S T}_{m}(\lambda)$, and $\mathbf{B}_{0 \mid n}(\lambda)\left(\lambda \in \mathcal{P}_{0 \mid n}\right)$ with $\mathcal{S} \mathcal{S} \mathcal{T}_{n}\left(\lambda^{\prime}\right)$, where $\lambda^{\prime}$ is the conjugate of $\lambda$. 
With this notation, we can recover several variations of the original Knuth correspondence from Theorem 3.11 (cf. [7]). If we put $n=v=0$ or $m=u=0$, then we have two kinds of Knuth correspondence, where the one is given by the column insertion of words and the other is given by the row insertion of words. If we put $m=v=0$ or $n=u=0$, then we obtain the dual Knuth correspondence. Similarly, one may also obtain other variations from the decomposition given in next section (see Theorem 4.5).

\subsection{Diagonal action on symmetric matrices}

Suppose that $m=u$ and $n=v$. Set

$$
\mathfrak{M}=\left\{A \in \mathbf{M} \mid A=A^{t}\right\},
$$

the set of all symmetric matrices in M. Let us consider the diagonal action of $\mathfrak{g l}_{m \mid n} \oplus$ $\mathfrak{g l}_{m \mid n}$ on $\mathfrak{M}$. That is, for $i \in I_{m \mid n}$ and $A \in \mathfrak{M}$, we define

$$
\begin{gathered}
\mathbf{e}_{i} A=e_{i} e_{i}^{*} A=e_{i}^{*} e_{i} A, \\
\mathbf{f}_{i} A=f_{i} f_{i}^{*} A=f_{i}^{*} f_{i} A .
\end{gathered}
$$

Note that $P_{1}(A)=P_{2}(A)$ for $A \in \mathfrak{M}(\operatorname{see}(3.5))$. Put wt $(A)=\operatorname{wt}\left(P_{1}(A)\right)=\operatorname{wt}\left(P_{2}(A)\right)$, $\varepsilon_{i}(A)=\max \left\{k \mid \mathbf{e}_{i}^{k} A \neq \mathbf{0}\right\}$, and $\varphi_{i}(A)=\max \left\{k \mid \mathbf{f}_{i}^{k} A \neq \mathbf{0}\right\}$ for $i \in I_{m \mid n}$ and $A \in \mathfrak{M}$. Then we have

Proposition 3.13. $\mathfrak{M}$ is a crystal graph for $\mathfrak{g l}_{m \mid n}$, which decomposes as follows;

$$
\mathfrak{M} \simeq \bigoplus_{\lambda \in \mathcal{P}_{m \mid n}} \mathbf{B}_{m \mid n}(\lambda)
$$

Proof: If $\mathbf{e}_{i} A \neq 0$ or $\mathbf{f}_{i} A \neq 0$ for $A \in \mathfrak{M}$ and $i \in I_{m \mid n}$, then

$$
\begin{gathered}
\left(\mathbf{e}_{i} A\right)^{t}=\left(e_{i} e_{i}^{*} A\right)^{t}=e_{i}^{*} e_{i} A^{t}=\mathbf{e}_{i} A \in \mathfrak{M}, \\
\left(\mathbf{f}_{i} A\right)^{t}=\left(f_{i} f_{i}^{*} A\right)^{t}=f_{i}^{*} f_{i} A^{t}=\mathbf{f}_{i} A \in \mathfrak{M} .
\end{gathered}
$$

Hence, $\mathbf{e}_{i}, \mathbf{f}_{i}: \mathfrak{M} \rightarrow \mathfrak{M} \cup\{\boldsymbol{0}\}$ are well-defined operators for $i \in I_{m \mid n}$.

For $A \in \mathfrak{M}$ and $i \in I_{m \mid n}$, we have $x_{i} A \neq \mathbf{0}$ if and only if $x_{i}^{*} A \neq \mathbf{0}(x=e, f)$ since $A$ is symmetric. Hence, by Lemma 3.6 , we have

$$
\begin{aligned}
& \mathbf{e}_{i} A \neq \mathbf{0} \Leftrightarrow P_{1}\left(e_{i} A\right) \neq \mathbf{0} \Leftrightarrow e_{i} P_{1}(A) \neq \mathbf{0}, \\
& \mathbf{f}_{i} A \neq \mathbf{0} \Leftrightarrow P_{1}\left(f_{i} A\right) \neq \mathbf{0} \Leftrightarrow f_{i} P_{1}(A) \neq \mathbf{0},
\end{aligned}
$$

for $i \in I_{m \mid n}$. This implies that $\mathfrak{M}$ is a $\mathfrak{g l}_{m \mid n}$-crystal.

Next, consider the decomposition of $\mathfrak{M}$. For $A \in \mathfrak{M}, A$ is $\mathfrak{g l}_{m \mid n}$-equivalent to $P_{1}(A)$. So each connected component in $\mathfrak{M}$ is generated by $A_{\lambda}$ for some $\lambda \in \mathcal{P}_{m \mid n}$ by Theorem Springer 
3.11. Since $A_{\lambda} \in \mathfrak{M}$ for $\lambda \in \mathcal{P}_{m \mid n}$, the set of all the highest weight elements in $\mathfrak{M}$ is equal to $\mathbf{M}_{\text {h.w. }}$.

Now, let us show that there is an interesting relation between the diagonal entries of a matrix in $\mathfrak{M}$ and the shape of the corresponding tableau (cf. [17]), and hence obtain a family of subcrystals of $\mathfrak{M}$, which also have nice decompositions. For $A=$ $\left(a_{b b^{\prime}}\right)_{b, b^{\prime} \in \mathbf{B}_{m \mid n}} \in \mathfrak{M}$, let

$$
\mathfrak{o}(A)=\left|\left\{b \in \mathbf{B}_{m \mid n}^{+} \mid a_{b b} \equiv 1(\bmod 2)\right\}\right|+\sum_{b \in \mathbf{B}_{m \mid n}^{-}} a_{b b} .
$$

Proposition 3.14. Let $\mathfrak{M}_{k}=\{A \in \mathfrak{M} \mid \mathfrak{o}(A)=k\}$ for $k \geq 0$. Then $\mathfrak{M}_{k}$ is a subcrystal of $\mathfrak{M}$, and decomposes as follows;

$$
\mathfrak{M}_{k} \simeq \bigoplus_{\substack{\lambda \in \mathcal{P}_{m \mid n} \\ o(\lambda)=k}} \mathbf{B}_{m \mid n}(\lambda)
$$

where $o(\lambda)$ is the number of odd parts in $\lambda$.

Proof: Fix $k \geq 0$. First, we will check that $\mathfrak{M}_{k}$ together with $\mathbf{0}$ is stable under $\mathbf{e}_{i}, \mathbf{f}_{i}$ $\left(i \in I_{m \mid n}\right)$, which implies that $\mathfrak{M}_{k}$ is a subcrystal of $\mathfrak{M}$.

Given $A=\left(a_{b b^{\prime}}\right) \in \mathfrak{M}_{k}$ and $i \in I_{m \mid n}$, suppose that $\mathbf{f}_{i} A \neq \mathbf{0}$. We assume that the diagonal entries of $A$ are changed under $\mathbf{f}_{i}$, equivalently under $f_{i}$ or $f_{i}^{*}$. Otherwise, it is clear that $\mathbf{f}_{i} A \in \mathfrak{M}_{k}$. For convenience, we write

$$
A[i]=\left(\begin{array}{cc}
a_{b b} & a_{b b^{\prime}} \\
a_{b^{\prime} b} & a_{b^{\prime} b^{\prime}}
\end{array}\right),
$$

where $b<b^{\prime}$ are the indices such that $\left\langle h_{i}, \epsilon_{b}\right\rangle,\left\langle h_{i}, \epsilon_{b^{\prime}}\right\rangle \neq 0$.

Case 1. $i=\bar{k},(1 \leq k \leq m-1)$. Consider

$$
A[i]=\left(\begin{array}{cc}
a_{\overline{k+1} \overline{k+1}} & a_{\overline{k+1} \bar{k}} \\
a_{\bar{k} \overline{k+1}} & a_{\bar{k} \bar{k}}
\end{array}\right)=\left(\begin{array}{cc}
a & b \\
b & c
\end{array}\right) .
$$

Suppose that $\left(f_{i} A\right)[i]=\left(\begin{array}{ccc}a-1 & b \\ b+1 & c\end{array}\right)$. This implies that with respect to $f_{i}$, we have $\epsilon^{(i)}(A)=\left(\ldots,-^{b},+^{a},-^{c},+^{b}, \ldots\right)$, where $a>c$ and the $i$-good + sign of $A$ appears in $+^{a}$ of $\epsilon^{(i)}(A)$. Note that $\epsilon^{(i)}\left(f_{i} A\right)=\left(\ldots,-{ }^{b},+^{a-1},-{ }^{c},+{ }^{b+1}, \ldots\right)$ with respect to $f_{i}^{*}$.

If $a \equiv c(\bmod 2)$, then we still have $a-1>c$, and the $i-\operatorname{good}+\operatorname{sign}$ of $f_{i} A$ with respect to $f_{i}^{*}$ appears in $+^{a-1}$, and we have

$$
\left(\mathbf{f}_{i} A\right)[i]=\left(f_{i}^{*} f_{i} A\right)[i]=\left(\begin{array}{cc}
a-2 & b+1 \\
b+1 & c
\end{array}\right) .
$$


If $a \not \equiv c(\bmod 2)$, then we have

$$
\left(\mathbf{f}_{i} A\right)[i]=\left(f_{i}^{*} f_{i} A\right)[i]=\left(\begin{array}{cc}
a-2 & b+1 \\
b+1 & c
\end{array}\right) \text { or }\left(\begin{array}{cc}
a-1 & b \\
b & c+1
\end{array}\right) .
$$

In any case, we have $\mathfrak{o}\left(\mathbf{f}_{i} A\right)=k$ and $\mathbf{f}_{i} A \in \mathfrak{M}_{k}$.

Next, suppose that $\left(f_{i} A\right)[i]=\left(\begin{array}{ll}a & b-1 \\ b & c+1\end{array}\right)$. Then we must have $\left(\mathbf{f}_{i} A\right)[i]=\left(\begin{array}{cc}a & b-1 \\ b-1\end{array}\right)$, and $\mathfrak{o}\left(\mathbf{f}_{i} A\right)=k$.

Case 2. $i=k,(1 \leq k \leq n-1)$. Consider

$$
A[i]=\left(\begin{array}{cc}
a_{k k} & a_{k k+1} \\
a_{k+1 k} & a_{k+1 k+1}
\end{array}\right)=\left(\begin{array}{cc}
a & b \\
b & c
\end{array}\right) .
$$

Suppose that $a>0$ and $\left(f_{i} A\right)[i]=\left(\begin{array}{ll}a-1 & b \\ b+1 & c\end{array}\right)$. Then with respect to $f_{i}$, we have $\epsilon^{(i)}(A)=\left(\ldots,+^{a},-{ }^{b},+^{b},-^{c}, \ldots\right)$, where the $i$-good + sign of $A$ appears in $+^{a}$. Since $\epsilon^{(i)}\left(f_{i} A\right)=\left(\ldots,+{ }^{a-1},-{ }^{b},+{ }^{b+1},-^{c}, \ldots\right)$ with respect to $f_{i}^{*}$, the $i$-good + sign of $f_{i} A$ appears in $+^{b+1}$, and we have

$$
\left(\mathbf{f}_{i} A\right)[i]=\left(f_{i}^{*} f_{i} A\right)[i]=\left(\begin{array}{cc}
a-1 & b \\
b & c+1
\end{array}\right)
$$

which implies that $\mathfrak{o}\left(\mathbf{f}_{i} A\right)=k$.

Next, suppose that $b>0$ and $\left(f_{i} A\right)[i]=\left(\begin{array}{cc}a & b-1 \\ b & c+1\end{array}\right)$. But, this can't happen since $\epsilon^{(i)}(A)=\left(\ldots,+^{a},-{ }^{b},+^{b},-^{c}, \ldots\right)$ with respect to $f_{i}$, and the pair $\left(-{ }^{b},+^{b}\right)$ cancels out.

Case 3. $i=0$. Consider

$$
A[0]=\left(\begin{array}{ll}
a_{\overline{1} \overline{1}} & a_{\overline{1} 1} \\
a_{1 \overline{1}} & a_{11}
\end{array}\right)=\left(\begin{array}{ll}
a & b \\
b & c
\end{array}\right) .
$$

Then we have

$$
\left(\mathbf{f}_{0} A\right)[0]=\left(f_{0}^{*} f_{0} A\right)[0]=\left(\begin{array}{cc}
a-2 & b+1 \\
b+1 & c
\end{array}\right) \text { or }\left(\begin{array}{cc}
a-1 & b \\
b & c+1
\end{array}\right)
$$

(it can't happen that $\left(\mathbf{f}_{0} A\right)[0]=\left(\begin{array}{ll}{ }_{b}-1 & b-1 \\ c+2\end{array}\right)$. In any case, we have $\mathfrak{o}\left(\mathbf{f}_{i} A\right)=k$.

Similarly, we can check that $\mathbf{e}_{i} \mathfrak{M}_{k} \subset \mathfrak{M}_{k} \cup\{\boldsymbol{0}\}$ for $i \in I_{m \mid n}$. Therefore, $\mathfrak{M}_{k}$ is a crystal graph for $\mathfrak{g l}_{m \mid n}$.

Next, we observe that for $\lambda=\left(\lambda_{k}\right)_{k \geq 1} \in \mathcal{P}_{m \mid n}$,

$$
\mathfrak{o}\left(A_{\lambda}\right)=\mid\left\{i \mid 1 \leq i \leq m, \lambda_{i} \text { is odd }\right\} \mid+\sum_{k \geq 0} \nu_{2 k+1},
$$


(see (3.8)). Since $v_{i}$ is the number of occurrences of $i$ in $\left(\lambda_{m+1}, \lambda_{m+2}, \ldots\right)$ (see (3.7)), it follows that $\mathfrak{o}\left(A_{\lambda}\right)$ is the number of odd parts in $\lambda$, say $o(\lambda)$, and hence $A_{\lambda} \in \mathfrak{M}_{k}$ if and only if $o(\lambda)=k$.

Corollary 3.15. Under the above hypothesis, we have $\mathfrak{M}=\bigoplus_{k \geq 0} \mathfrak{M}_{k}$, and in particular,

$$
\mathfrak{M}_{0} \simeq \bigoplus_{\substack{\lambda \in \mathcal{P}_{m \mid n} \\ \lambda: \text { even }}} \mathbf{B}_{m \mid n}(\lambda)
$$

Proof: It follows from the fact that $\mathfrak{o}\left(A_{\lambda}\right)=0$ if and only if $\lambda$ is even (that is, each part of $\lambda$ is even).

Remark 3.16.

(1) A special case of Proposition 3.14 was first observed in [17]. Let us give a brief explanation. Put $m=0$ in Proposition 3.14. We identify $\mathbf{B}_{0 \mid n}$ with $\mathcal{A}_{n}$ (see Remark 3.12 ), and $\mathbf{B}_{0 \mid n}(\lambda)$ with $\mathcal{S} \mathcal{S} \mathcal{T}_{n}\left(\lambda^{\prime}\right)$. Hence, the set of all $n \times n$ symmetric matrices of non-negative integers with $\operatorname{tr}(A)=k$, is in one-to-one correspondence with $\bigsqcup_{o\left(\lambda^{\prime}\right)=k} \mathcal{S S T}_{n}(\lambda)$ (Theorem 4 [17]) where the union is given over all partitions with the number of odd columns $k$.

(2) If we consider the characters of the decompositions in Proposition 3.13 and Corollary 3.15, then we obtain the following identities (cf. [18, 19]);

$$
\frac{\prod_{b<b^{\prime},|b| \neq\left|b^{\prime}\right|}\left(1+x_{b} x_{b^{\prime}}\right)}{\prod\left(1-x_{b}\right) \prod_{b<b^{\prime},|b|=\left|b^{\prime}\right|}\left(1-x_{b} x_{b^{\prime}}\right)}=\sum_{\lambda \in \mathcal{P}_{m \mid n}} h s_{\lambda}(x),
$$

and

$$
\frac{\prod_{b<b^{\prime},|b| \neq\left|b^{\prime}\right|}\left(1+x_{b} x_{b^{\prime}}\right)}{\prod_{|b|=0}\left(1-x_{b}^{2}\right) \prod_{b<b^{\prime},|b|=\left|b^{\prime}\right|}\left(1-x_{b} x_{b^{\prime}}\right)}=\sum_{\substack{\lambda \in \mathcal{P}_{m \mid n} \\ \lambda: \text { even }}} h s_{\lambda}(x),
$$

where $b, b^{\prime} \in \mathbf{B}_{m \mid n}$.

\section{Dual construction}

In this section, we discuss a bicrystal graph associated to the super exterior algebra $\Lambda\left(\mathbb{C}^{m \mid n} \otimes \mathbb{C}^{u \mid v}\right)$, and its explicit decomposition.

Suppose that $m, n, u, v$ are non-negative integers such that $m+n, u+v>0$. We set

$$
\begin{aligned}
\mathbf{M}_{m|n, u| v}^{\sharp}= & \left\{A=\left(a_{b b^{\prime}}\right)_{b \in \mathbf{B}_{m \mid n}, b^{\prime} \in \mathbf{B}_{u \mid v} \mid}\right. \\
& \text { (1) } \left.a_{b b^{\prime}} \in \mathbb{Z}_{\geq 0}, \text { (2) } a_{b b^{\prime}} \leq 1 \text { if }|b|=\left|b^{\prime}\right|\right\} .
\end{aligned}
$$


For convenience, we write $\mathbf{M}^{\sharp}=\mathbf{M}_{m|n, u| v}^{\sharp}$.

As in the case of $\mathbf{M}$, we identify a matrix in $\mathbf{M}^{\sharp}$ with a biword given by reading the row and column indices of non-zero entries of the matrix with respect to a linear ordering. First, we let

$$
\begin{aligned}
\Omega_{m|n, u| v}^{\sharp}= & \Omega^{\sharp}=\left\{(\mathbf{i}, \mathbf{j}) \in \mathcal{W}_{m \mid n} \times \mathcal{W}_{u \mid v} \mid\right. \\
& \text { (1) } \mathbf{i}=i_{1} \ldots i_{r} \text { and } \mathbf{j}=j_{1} \ldots j_{r} \text { for some } r \geq 0, \\
& \text { (2) }\left(i_{1}, j_{1}\right) \preceq \cdots \preceq\left(i_{r}, j_{r}\right), \\
& \text { (3) } \left.\left|i_{k}\right|=\left|j_{k}\right| \text { implies }\left(i_{k}, j_{k}\right) \neq\left(i_{k \pm 1}, j_{k \pm 1}\right)\right\},
\end{aligned}
$$

where for $(i, j)$ and $(k, l) \in \mathbf{B}_{m \mid n} \times \mathbf{B}_{u \mid v}$, the linear ordering $\prec$ is given by

$$
(i, j) \prec(k, l) \Leftrightarrow \begin{cases}(j<l) & \text { or, } \\ \left(j=l \in \mathbf{B}_{u \mid v}^{+}, \text {and } i<k\right) & \text { or, } \\ \left(j=l \in \mathbf{B}_{u \mid v}^{-}, \text {and } i>k\right) & \end{cases}
$$

We define $e_{i}, f_{i}: \Omega^{\sharp} \rightarrow \Omega^{\sharp} \cup\{\mathbf{0}\}\left(i \in I_{m \mid n}\right)$ by

$$
e_{i}(\mathbf{i}, \mathbf{j})=\left(e_{i} \mathbf{i}, \mathbf{j}\right), \quad f_{i}(\mathbf{i}, \mathbf{j})=\left(f_{i} \mathbf{i}, \mathbf{j}\right)
$$

for $(\mathbf{i}, \mathbf{j}) \in \Omega^{\sharp}$. Set $w \mathrm{t}(\mathbf{i}, \mathbf{j})=\operatorname{wt}(\mathbf{i}), \varepsilon_{i}(\mathbf{i}, \mathbf{j})=\varepsilon_{i}(\mathbf{i})$ and $\varphi_{i}(\mathbf{i}, \mathbf{j})=\varphi_{i}(\mathbf{i})\left(i \in I_{m \mid n}\right)$. Then it is easy to see that $\Omega^{\sharp}$ is a crystal graph for $\mathfrak{g l}_{m \mid n}$ (cf. Lemma 3.1).

For $(\mathbf{i}, \mathbf{j}) \in \Omega_{m|n, u| v}^{\sharp}$, we define $A(\mathbf{i}, \mathbf{j})=\left(a_{b b^{\prime}}\right)$ to be a matrix in $\mathbf{M}^{\sharp}$, where $a_{b b^{\prime}}$ is the number of $k$ 's such that $\left(i_{k}, j_{k}\right)=\left(b, b^{\prime}\right)$ for $b \in \mathbf{B}_{m \mid n}$ and $b^{\prime} \in \mathbf{B}_{u \mid v}$. Then, the map $(\mathbf{i}, \mathbf{j}) \mapsto A(\mathbf{i}, \mathbf{j})$ is a bijection between $\Omega_{m|n, u| v}^{\sharp}$ and $\mathbf{M}^{\sharp}$. Hence, $\mathbf{M}^{\sharp}$ is a crystal graph for $\mathfrak{g l}_{m \mid n}$ with this identification.

Next, we introduce $\left(\Omega_{m|n, u| v}^{\sharp}\right)^{*}$ to define a $\mathfrak{g l}_{u \mid v}$-crystal structure on $\mathbf{M}^{\sharp}$. Recall that in Section 3, a biword in $\Omega^{*}$ was obtained by reading the row and column indices of the non-zero entries in the transpose of a given matrix with respect to the same lexicographic ordering used in $\Omega$. But in the case of $\mathbf{M}^{\sharp}$, we need another linear ordering. That is, we set

$$
\begin{aligned}
\left(\Omega_{m|n, u| v}^{\sharp}\right)^{*}=\left(\Omega^{\sharp}\right)^{*}= & \left\{(\mathbf{k}, \mathbf{1}) \in \mathcal{W}_{m \mid n} \times \mathcal{W}_{u \mid v} \mid\right. \\
& \text { (1) } \mathbf{k}=k_{1} \ldots k_{r} \text { and } \mathbf{1}=l_{1} \ldots l_{r} \text { for some } r \geq 0, \\
& \text { (2) }\left(k_{1}, l_{1}\right) \preceq^{\prime} \ldots \preceq^{\prime}\left(k_{r}, l_{r}\right), \\
& \text { (3) } \left.\left|k_{t}\right|=\left|l_{t}\right| \text { implies }\left(k_{t}, l_{t}\right) \neq\left(k_{t \pm 1}, l_{t \pm 1}\right)\right\},
\end{aligned}
$$


where for $(i, j)$ and $(k, l) \in \mathbf{B}_{m \mid n} \times \mathbf{B}_{u \mid v}$, the linear ordering $\prec^{\prime}$ is given by

$$
(i, j) \prec^{\prime}(k, l) \Leftrightarrow \begin{cases}(i>k) & \text { or, } \\ \left(i=k \in \mathbf{B}_{u \mid v}^{+}, \text {and } j<l\right) & \text { or, } \\ \left(i=k \in \mathbf{B}_{u \mid v}^{-}, \text {and } j>l\right) . & \end{cases}
$$

Clearly, we have a bijection $(\mathbf{k}, \mathbf{1}) \mapsto A(\mathbf{k}, \mathbf{1})$ from $\left(\Omega^{\sharp}\right)^{*}$ to $\mathbf{M}^{\sharp}$.

Similarly, we define $e_{j}^{*}, f_{j}^{*}:\left(\Omega^{\sharp}\right)^{*} \rightarrow\left(\Omega^{\sharp}\right)^{*} \cup\{\mathbf{0}\}\left(j \in I_{u \mid v}\right)$ by

$$
e_{j}^{*}(\mathbf{k}, \mathbf{1})=\left(\mathbf{k}, e_{j} \mathbf{1}\right), \quad f_{j}^{*}(\mathbf{k}, \mathbf{1})=\left(\mathbf{k}, f_{j} \mathbf{1}\right)
$$

for $(\mathbf{k}, \mathbf{1}) \in\left(\Omega^{\sharp}\right)^{*}$. Set $\operatorname{wt}^{*}(\mathbf{k}, \mathbf{1})=\operatorname{wt}(\mathbf{1}), \varepsilon_{j}^{*}(\mathbf{k}, \mathbf{1})=\varepsilon_{j}(\mathbf{1})$ and $\varphi_{j}^{*}(\mathbf{k}, \mathbf{1})=\varphi_{j}(\mathbf{1})(j \in$ $I_{u \mid v}$ ). Then $\left(\Omega^{\sharp}\right)^{*}$ is a crystal graph for $\mathfrak{g l}_{u \mid v}$ (cf. Lemma 3.1), and hence so is $\mathbf{M}^{\sharp}$.

Example 4.1. Suppose that $m|n=u| v=2 \mid 2$ and

$$
A=\left(\begin{array}{ll|ll}
1 & 1 & 0 & 0 \\
0 & 0 & 2 & 1 \\
\hline 0 & 1 & 1 & 0 \\
2 & 0 & 0 & 0
\end{array}\right) \in \mathbf{M}^{\sharp} .
$$

Then $A=A(\mathbf{i}, \mathbf{j})=A(\mathbf{k}, \mathbf{1})$ for $(\mathbf{i}, \mathbf{j}) \in \Omega^{\sharp}$ and $(\mathbf{k}, \mathbf{1}) \in\left(\Omega^{\sharp}\right)^{*}$, where

$$
\begin{array}{ll}
\mathbf{i}=\overline{2} 22 \overline{2} 11 \overline{1} \overline{1} \overline{1}, & \mathbf{k}=2211 \overline{1} \overline{1} \overline{1} \overline{2} \\
\mathbf{j}=\overline{2} \overline{2} \overline{2} \overline{1} \overline{1} 1112, & \mathbf{1}=\overline{2} \overline{2} 1 \overline{1} 112 \overline{2} \overline{1} .
\end{array}
$$

For $A \in \mathbf{M}^{\sharp}$, we have $A=A(\mathbf{i}, \mathbf{j})=A(\mathbf{k}, \mathbf{1})$ for unique $(\mathbf{i}, \mathbf{j}) \in \Omega^{\sharp}$ and $(\mathbf{k}, \mathbf{1}) \in$ $\left(\Omega^{\sharp}\right)^{*}$. Then we define

$$
\pi^{\sharp}(A)=\left(P_{1}^{\sharp}(A), P_{2}^{\sharp}(A)\right)=(P(\mathbf{i}), P(\mathbf{1})) .
$$

By definition, we have $A \simeq_{\mathfrak{g l}_{m \mid n}} P_{1}^{\sharp}(A)$, and $A \simeq_{\mathfrak{g l}_{u \mid v}} P_{2}^{\sharp}(A)$.

Now, we have following analogue of Lemma 3.4 and Proposition 3.7;

Proposition 4.2. $\mathbf{M}^{\sharp}$ is a $\left(\mathfrak{g l}_{m \mid n}, \mathfrak{g l}_{u \mid v}\right)$-bicrystal, and for each connected component $\mathbf{C}$ in $\mathbf{M}^{\sharp}, \pi^{\sharp}$ gives the following isomorphism of $\left(\mathfrak{g l}_{m \mid n}, \mathfrak{g l}_{u \mid v}\right)$-bicrystals;

$$
\pi^{\sharp}: \mathbf{C} \longrightarrow \mathbf{B}_{m \mid n}(\lambda) \times \mathbf{B}_{u \mid v}(\mu)
$$

for some $\lambda \in \mathcal{P}_{m \mid n}$ and $\mu \in \mathcal{P}_{u \mid v}$. 
Proof: For $A \in \mathbf{M}^{\sharp}$, let $A^{\rho}$ be the matrix given as a clock-wise rotation of $A$ by $90^{\circ}$ (see Fig. 2). The map $A \mapsto A^{\rho}$ gives a one-to-one correspondence from $\mathbf{M}_{m|n, u| v}^{\sharp}$ to $\mathbf{M}_{u|v, n| m}$. Moreover, we have

$$
\begin{aligned}
& \left(e_{\bar{k}} A\right)^{\rho}=f_{k}^{*} A^{\rho}, \quad\left(f_{\bar{k}} A\right)^{\rho}=e_{k}^{*} A^{\rho}, \quad(1 \leq k \leq m-1), \\
& \left(e_{l} A\right)^{\rho}=f_{\bar{l}}^{*} A^{\rho}, \quad\left(f_{l} A\right)^{\rho}=e_{l}^{*} A^{\rho}, \quad(1 \leq l \leq n-1), \\
& \left(e_{0} A\right)^{\rho}=f_{0}^{*} A^{\rho}, \quad\left(f_{0} A\right)^{\rho}=e_{0}^{*} A^{\rho},
\end{aligned}
$$

and $\left(x_{j}^{*} A\right)^{\rho}=x_{j} A^{\rho}$ for $j \in I_{u \mid v}$ and $x=e, f$. For example, let us consider $\left(e_{\bar{k}} A\right)^{\rho}=$ $f_{k}^{*} A^{\rho}$. If $A=\left(a_{b b^{\prime}}\right)$ and $e_{\bar{k}} A \neq \mathbf{0}$, then $e_{\bar{k}} A=A-E_{\bar{k} b^{\prime}}+E_{\overline{k+1} b^{\prime}}$ for some $b^{\prime} \in \mathbf{B}_{u \mid v}$, where $E_{b b^{\prime}}\left(b \in \mathbf{B}_{m \mid n}, b^{\prime} \in \mathbf{B}_{u \mid v}\right)$ denotes the elementary matrix corresponding to the $\left(b, b^{\prime}\right)$-position. This implies that the $\bar{k}$-good-sign of $A$ occurs at $a_{\bar{k} b^{\prime}}$. On the other hand, in $A^{\rho}, a_{\bar{k} b^{\prime}}$ is located at $\left(b^{\prime}, k\right)$-position, and it gives the $k$-good $+\operatorname{sign}$ of $A^{\rho}$ with respect to $f_{k}^{*}$ (see Section 2.2). Hence, we have

$$
f_{k}^{*} A^{\rho}=A^{\rho}-E_{b^{\prime} k}+E_{b^{\prime} k+1}=\left(A-E_{\bar{k} b^{\prime}}+E_{\overline{k+1} b^{\prime}}\right)^{\rho}=\left(e_{\bar{k}} A\right)^{\rho} .
$$

Other cases can be checked similarly.

By Lemma 3.4, $e_{i}, f_{i}\left(i \in I_{m \mid n}\right)$ commute with $e_{j}^{*}, f_{j}^{*}\left(j \in I_{u \mid v}\right)$ on $\mathbf{M}^{\sharp}$. Hence, $\mathbf{M}^{\sharp}$ is a $\left(\mathfrak{g l}_{m \mid n}, \mathfrak{g l}_{u \mid v}\right)$-bicrystal.

Applying the same arguments in Lemma 3.5, 3.6 and Proposition 3.7, we conclude that each connected component of $\mathbf{M}^{\sharp}$ is isomorphic to $\mathbf{B}_{m \mid n}(\lambda) \times \mathbf{B}_{u \mid v}(\mu)$ for some $\lambda \in \mathcal{P}_{m \mid n}$ and $\mu \in \mathcal{P}_{u \mid v}$.

Example 4.3. Let $A$ be the matrix given in Example 4.1. Then we have

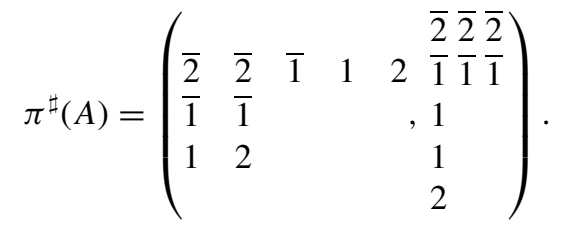

$$
\begin{aligned}
& \left(\begin{array}{lll|lll}
0 & 1 & 1 & 0 & 0 & 2 \\
1 & 0 & 0 & 2 & 1 & 0 \\
\hline 3 & 0 & 1 & 1 & 0 & 1 \\
0 & 2 & 0 & 0 & 0 & 0
\end{array}\right)^{\rho}=\left(\begin{array}{ll|ll}
0 & 3 & 1 & 0 \\
2 & 0 & 0 & 1 \\
0 & 1 & 0 & 1 \\
\hline 0 & 1 & 2 & 0 \\
0 & 0 & 1 & 0 \\
0 & 1 & 0 & 2
\end{array}\right)
\end{aligned}
$$

Fig. 2 A clock-wise rotation of $A$ by $90^{\circ}$ 
$\mathbf{M}_{\text {h.w. }}^{\sharp}=\left\{A \in \mathbf{M}^{\sharp} \mid \pi^{\sharp}(A)=\left(H_{m \mid n}^{\lambda}, H_{u \mid v}^{\mu}\right)\right.$ for some $\left.(\lambda, \mu) \in \mathcal{P}_{m \mid n} \times \mathcal{P}_{u \mid v}\right\}$,

which is the set of all the highest weight elements in $\mathbf{M}^{\sharp}$.

Suppose that $\lambda=\left(\lambda_{k}\right)_{k \geq 1} \in \mathcal{P}_{m \mid n} \cap \mathcal{P}_{v \mid u}$ is given, and let $\lambda^{\prime}=\left(\lambda_{k}^{\prime}\right)_{k \geq 1}$ be the conjugate of $\lambda$. We put $\ell=\lambda_{m+1}$. We define $A_{\lambda}^{\sharp}=\left(a_{b b^{\prime}}^{\sharp}\right) \in \mathbf{M}^{\sharp}$ as follows;

Case $1 . \ell \leq u$.

(1) for $0 \leq k<m, 0 \leq l<u$,

$$
a_{\overline{m-k}}^{\sharp} \frac{1-l}{u-l}= \begin{cases}1, & \text { if } \lambda_{k+1} \geq l+1, \\ 0, & \text { otherwise. }\end{cases}
$$

(2) for $0 \leq k<m, 1 \leq t \leq v$,

$$
a_{\overline{m-k} t}^{\sharp}= \begin{cases}\mu_{t}, & \text { if } k+1=t, \\ 0, & \text { otherwise, }\end{cases}
$$

where $\mu_{t}=\max \left(\lambda_{t}-u, 0\right)$ for $t \geq 1$.

(3) for $1 \leq s \leq n, 0 \leq l<u$,

$$
a_{s \frac{u-l}{\sharp}}^{\sharp}= \begin{cases}v_{s+l}, & \text { if } 1 \leq s+l \leq \ell, \\ 0, & \text { otherwise, }\end{cases}
$$

where $v_{1}, \ldots, v_{\ell}$ are given in (3.7).

(4) for $1 \leq s \leq n, 1 \leq t \leq v, a_{s t}^{\sharp}=0$.

Note that $A_{\lambda}^{\sharp}$ is of the following form;

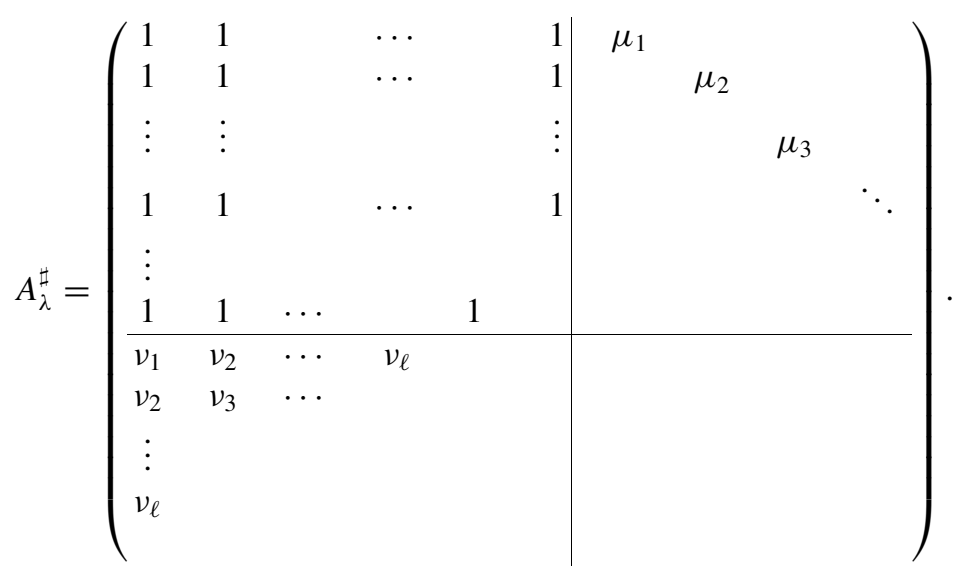

Case 2. $u<\ell \leq n$. In this case, we can check that $\lambda_{m} \geq u$ and $v>m$. 
(1') for $0 \leq k<m, 0 \leq l<u$,

$$
a_{\overline{m-k}}^{\sharp} \frac{}{u-l}=1 .
$$

(2') for $0 \leq k<m, 1 \leq t \leq v$,

$$
a_{\overline{m-k} t}^{\sharp}= \begin{cases}\mu_{t}, & \text { if } k+1=t, \\ 0, & \text { otherwise, }\end{cases}
$$

where $\mu_{t}=\lambda_{t}-u$ for $1 \leq t \leq m$.

(3') for $1 \leq s \leq n, 0 \leq l<u$,

$$
a_{s \overline{u-l}}^{\sharp}= \begin{cases}v_{s+l}, & \text { if } 1 \leq s+l \leq \ell, \\ 0, & \text { otherwise, }\end{cases}
$$

where $v_{1}, \ldots, v_{\ell}$ are given in (3.7).

(4') for $1 \leq s<\ell, m<t \leq v$,

$$
a_{s t}^{\sharp}= \begin{cases}1, & \text { if } t \geq m \text { and } t \leq \lambda_{u+s}^{\prime}, \\ 0, & \text { otherwise. }\end{cases}
$$

Then $A_{\lambda}^{\sharp}$ is of the following form;

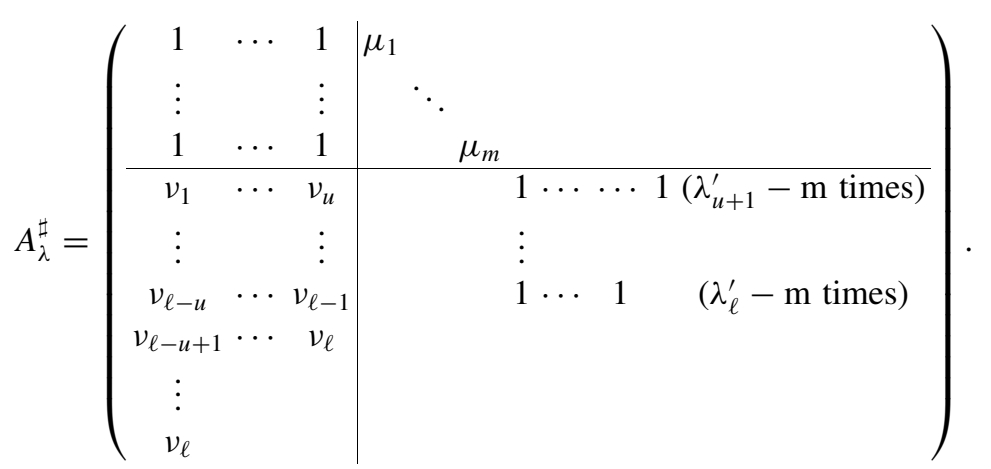

Note that $\lambda_{u+k}^{\prime}-m=v_{u+k}+\cdots+v_{\ell}$ for $1 \leq k \leq \ell-u$.

In both cases, we can check by Schensted's algorithm that

$$
\pi^{\sharp}\left(A_{\lambda}^{\sharp}\right)=\left(H_{m \mid n}^{\lambda}, H_{u \mid v}^{\lambda^{\prime}}\right) .
$$


Example 4.4. Suppose that $m|n=3| 2$ and $u|v=4| 3$. Let $\lambda=(7,6,2,2,1,1)$. Then

$$
A_{\lambda}^{\sharp}=\left(\begin{array}{llll|lll}
1 & 1 & 1 & 1 & 3 & 0 & 0 \\
1 & 1 & 1 & 1 & 0 & 2 & 0 \\
1 & 1 & 0 & 0 & 0 & 0 & 0 \\
\hline 2 & 1 & 0 & 0 & 0 & 0 & 0 \\
1 & 0 & 0 & 0 & 0 & 0 & 0
\end{array}\right),
$$

and

$$
\pi^{\sharp}\left(A_{\lambda}^{\sharp}\right)=\left(\begin{array}{lllllllllllll}
\overline{3} & \overline{3} & \overline{3} & \overline{3} & \overline{3} & \overline{3} & \overline{3} & \overline{3} & \overline{3} & \overline{3} & \overline{3} & & \\
\overline{2} & \overline{2} & \overline{2} & \overline{2} & \overline{2} & \overline{2} & \overline{2} & \overline{2} & & & & \\
\overline{1} & \overline{1} & & & & & & \overline{1} & \overline{1} & & & & \\
1 & 2 & & & & & & & & & \\
1 & & & & & & 1 & 2 & & & & \\
1 & & & & & 1 & 2 & & & & \\
& & & & & & 1 & & & &
\end{array}\right) .
$$

Now, we can characterize all the highest weight elements in $\mathbf{M}^{\sharp}$.

Theorem 4.5 (cf. [6]). We have

$$
\mathbf{M}_{\text {h.w. }}^{\sharp}=\left\{A_{\lambda}^{\sharp} \mid \lambda \in \mathcal{P}_{m \mid n} \cap \mathcal{P}_{v \mid u}\right\},
$$

and hence the following isomorphism of $\left(\mathfrak{g l}_{m \mid n}, \mathfrak{g l}_{u \mid v}\right)$-bicrystals;

$$
\pi^{\sharp}: \mathbf{M}^{\sharp} \longrightarrow \bigoplus_{\lambda \in \mathcal{P}_{m \mid n} \cap \mathcal{P}_{v \mid u}} \mathbf{B}_{m \mid n}(\lambda) \times \mathbf{B}_{u \mid v}\left(\lambda^{\prime}\right) .
$$

Proof: Since the proof is similar to that of Theorem 3.11, let us give a brief sketch of it, and leave the details to the reader.

Suppose that $A=\left(a_{b b^{\prime}}\right) \in \mathbf{M}_{\text {h.w. }}^{\sharp}$ is given, and $A=A(\mathbf{i}, \mathbf{j})=A(\mathbf{k}, \mathbf{1})$ for $(\mathbf{i}, \mathbf{j}) \in$ $\Omega^{\sharp},(\mathbf{k}, \mathbf{1}) \in\left(\Omega^{\sharp}\right)^{*}$. We claim that $A=A_{\lambda}^{\sharp}$ for some $\lambda \in \mathcal{P}_{m \mid n} \cap \mathcal{P}_{v \mid u}$ (see (4.8) and (4.9)). As in Theorem 3.11, we will also use the notations $\mathbf{i}=\mathbf{i}_{u} \mathbf{i}_{u-1} \cdots \mathbf{i}_{v-1} \mathbf{i}_{v}$ and $\mathbf{1}=\mathbf{1}_{n} \cdots \mathbf{1}_{\bar{m}}$, which are defined in a similar way. As in Theorem 3.11, we will divide $A$ into several submatrices and prove that each submatrix is of the form given in (4.8) or (4.9).

Step 1. First, we can check that if $a_{s \bar{k}}=0$ for all $1 \leq s \leq n$ and $1 \leq k \leq u$, then $a_{b b^{\prime}}=0$ for all $b \in \mathbf{B}_{m \mid n}^{-}$and $b^{\prime} \in \mathbf{B}_{u \mid v}$ (recall (H) in the proof of Theorem 3.11). Suppose that $a_{s \bar{k}} \neq 0$ for some $1 \leq s \leq n$ and $1 \leq k \leq u$. Let $\ell$ be the maximum row index $(1 \leq \ell \leq n)$ satisfying this condition. Put $s_{0}=\max \{\ell-u, 0\}+1$. Since $P\left(\mathbf{1}_{n} \ldots \mathbf{1}_{s}\right)$ satisfies $(\mathrm{H})$ and $e_{s} A=e_{k}^{*} A=\mathbf{0}$ for $s_{0} \leq s \leq \ell-1$ and $1 \leq k \leq u-$ 
1, it follows that the submatrix $\left(a_{s b^{\prime}}\right)_{s_{0} \leq s \leq n, b^{\prime} \in \mathbf{B}_{u \mid v}}$ is of the form given in (4.8) or (4.9) for some $v_{s}\left(s_{0} \leq s \leq \ell\right)$.

Step 2. If we consider $P\left(\mathbf{i}_{\bar{u}} \ldots \mathbf{i}_{\overline{1}}\right)$, then by $(\mathrm{H})$ and $e_{\bar{k}} A=e_{\bar{l}}^{*} A=\mathbf{0}(1 \leq k \leq m-1$, $1 \leq l \leq u-1)$, we see that the submatrix $\left(a_{b b^{\prime}}\right)_{b \in \mathbf{B}_{m \mid n}^{+}, b^{\prime} \in \mathbf{B}_{u \mid v}^{+}}$is of the form given in (4.8) or (4.9) (cf. Step 3 and Step 4 in Theorem 3.11).

Step 3. If $\ell \leq u$, then we can check that the submatrix $\left(a_{b t}\right)_{b \in \mathbf{B}_{m \mid n}^{+}, 1 \leq t \leq v}$ is of the form given in (4.8) for some $\mu_{t}(1 \leq t \leq v)$, since $P\left(\mathbf{i}_{\bar{u}} \ldots \mathbf{i}_{1} \mathbf{i}_{1} \ldots \mathbf{i}_{t}\right)(1 \leq t \leq v)$ and $P\left(\mathbf{1}_{n} \ldots \mathbf{1}_{1} \mathbf{i}_{\overline{1}} \ldots \mathbf{i}_{\bar{k}}\right)(1 \leq k \leq m)$ satisfy $(\mathrm{H})$, and and $e_{j}^{*} A=\mathbf{0}$ for $1 \leq j \leq v-1$.

Hence, if $\ell \leq u$, then we conclude that $A=A_{\lambda}^{\sharp}$ for some $\lambda \in \mathcal{P}_{m \mid n} \cap \mathcal{P}_{v \mid u}$ such that $\lambda_{m+1} \leq u$ (see (4.8)). From now on, we assume that $\ell>u$.

Step 3'. Similar to Step 3, we can check that (i) the submatrix $\left(a_{b t}\right)_{b \in \mathbf{B}_{m \mid n}^{+}, 1 \leq t \leq v}$ is of the form given in (4.9) for some $\mu_{t}(1 \leq t \leq m)$, and (ii) $a_{s t}=0\left(1 \stackrel{m}{\leq} \leq s_{0}\right.$, $1 \leq t \leq m)$, since the first $m$ rows of $P\left(\mathbf{i}_{\bar{u}} \ldots \mathbf{i}_{\overline{1}} \mathbf{i}_{1} \ldots \mathbf{i}_{t}\right)(1 \leq t \leq m)$, which satisfies $(\mathrm{H})$, forms a highest weight tableau and $e_{j}^{*} A=\mathbf{0}$ for $1 \leq j \leq v-1$.

Step 4. By similar argument as Step 4 in Theorem 3.11, we can check that if $a_{s t}=0$ for $1 \leq s<\ell-u$ and $m<t<v$, then $a_{s t+1}=a_{s+1 t}=0$.

Step 5. If we consider $P\left(\mathbf{1}_{n} \ldots \mathbf{1}_{s_{0}}\right)$, then by $(\mathrm{H}) v_{\ell}$ is no less than $\sum_{m<t \leq v} a_{\ell-u t}$ (the number of 1's in $a_{\ell-u t}$ for $m<t \leq v$ ). On the other hand, $e_{\ell-u} A=\mathbf{0}$ implies that $\nu_{\ell} \leq \sum_{m<t \leq v} a_{\ell-u t}$, and hence they are equal. Next, since $e_{\ell-u} A=\mathbf{0}$ and $e_{1}^{*} A=\mathbf{0}$, we have $a_{\ell-u \overline{1}}=a_{\ell-u+1}$, and similarly $a_{\ell-u \bar{k}}=a_{\ell-u+1} \overline{k+1}$ for $1 \leq k \leq u-1$. Continuing this argument, we conclude that $a_{s \overline{1}}=\sum_{m<t \leq v} a_{s-1 t}-\sum_{m<t \leq v} a_{s t}$ for $2 \leq s \leq \ell-u+1$, and $a_{s \bar{k}}=a_{s+1} \overline{k+1}$ for $1 \leq s \leq \ell-u$ and $1 \leq k \leq u-1$. If we put $v_{s}=a_{s \bar{u}}$ for $1 \leq s \leq \ell-u$, then $\sum_{m<t \leq v} a_{s t}=v_{u+s}+\cdots+v_{\ell}$ for $1 \leq$ $s \leq \ell-u$, and the submatrix $\left(a_{s b^{\prime}}\right)_{1 \leq s \leq \ell-u b^{\prime} \in \mathbf{B}_{u \mid v}}$ is of the form given in (4.9). Finally, we can check that $\mu_{m} \geq \ell-u$ since $e_{m}^{*} A=\mathbf{0}$.

Therefore, we conclude that $A=A_{\lambda}^{\sharp}$ for some $\lambda \in \mathcal{P}_{m \mid n} \cap \mathcal{P}_{v \mid u}$ such that $\lambda_{m+1}>u$ (see (4.9)). This completes the proof.

In terms of characters, we also recover the dual Cauchy identity of hook Schur functions;

$$
\frac{\prod_{|b|=\left|b^{\prime}\right|}\left(1+x_{b} y_{b^{\prime}}\right)}{\prod_{|b| \neq\left|b^{\prime}\right|}\left(1-x_{b} y_{b^{\prime}}\right)}=\sum_{\lambda \in \mathcal{P}_{m \mid n} \cap \mathcal{P}_{v \mid u}} h s_{\lambda}(x) h s_{\lambda^{\prime}}(y),
$$

where $b \in \mathbf{B}_{m \mid n}$ and $b^{\prime} \in \mathbf{B}_{u \mid v}$.

\section{Semi-infinite construction}

Let $\mathfrak{g}$ be a contragredient Lie superalgebra of infinite rank whose associated Dynkin diagram is given by

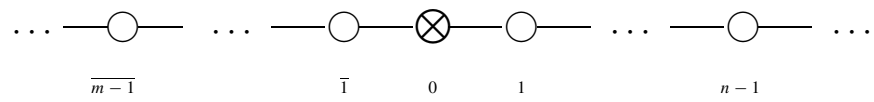

(see [11]). Then for all $m, n \geq 1$, there is a natural embedding of $\mathfrak{g l}_{m \mid n}$ into $\mathfrak{g}$. Note that $\mathfrak{g}$ is not equal to $\mathfrak{g l}_{\infty \mid \infty}$ in the sense of [12], but a proper subalgebra of it. In this section, Springer 
we study crystal graphs for $\mathfrak{g}$ which are generated by highest weight elements. Since a tensor power of the $\mathfrak{g}$-crystal associated to the natural representation does not have a highest weight element, we introduce a $\mathfrak{g}$-crystal $\mathscr{F}$ consisting of semi-infinite words, which is analogous to the crystal graph for $\mathfrak{g l}_{\infty}$ associated to a level one fermionic Fock space representation (cf. [10]). We will show that each connected component of a tensor power of $\mathscr{F}$ can be realized as the set of semi-infinite semistandard tableaux, which is generated by a highest weight vector. Then by using the methods developed in the previous sections, we give an explicit decomposition of $\mathscr{F} \otimes u(u \geq 2)$ as a $\left(\mathfrak{g}, \mathfrak{g l}_{u}\right)$-bicrystal.

\subsection{Crystal graphs of semi-infinite words}

We may naturally define a crystal graph for $\mathfrak{g}$ by taking $m$ and $n$ to infinity in Definition 2.1. Set

$$
\mathbf{B}=\{\cdots<\bar{m}<\cdots<\overline{1}<1<\cdots<n<\cdots\}
$$

and $\mathbf{B}^{+}$(resp. $\mathbf{B}^{-}$) denotes the set of elements with degree 0 (resp. 1) in $\mathbf{B}$. The index set is given by

$$
I=\{\ldots, \bar{m}, \ldots, \overline{1}, 0,1, \ldots, n, \ldots\}
$$

Then the simple root $\alpha_{i}$ and the simple coroot $h_{i}(i \in I)$ are defined in the same way. But, instead of $\bigoplus_{b \in \mathbf{B}} \mathbb{Z} \epsilon_{b}$, we use

$$
P=\mathbb{Z} \Lambda \oplus \bigoplus_{b \in \mathbf{B}} \mathbb{Z} \epsilon_{b}
$$

as the weight lattice of $\mathfrak{g}$, where $\Lambda=\Lambda_{0} \in\left(\bigoplus_{i \in I} \mathbb{Z} h_{i}\right)^{*}$ is the fundamental weight such that $\left\langle h_{i}, \Lambda\right\rangle=\delta_{0 i}$ for $i \in I$. Note that $\mathbf{B}$ is the crystal graph for $\mathfrak{g}$ associated to the natural representation of $\mathfrak{g}$.

Now, we define $\mathscr{F}$ to be the set of semi-infinite words $w=\cdots w_{3} w_{2} w_{1}$ with letters in $\mathbf{B}$ such that

(1) $w_{i+1} \leq w_{i}$ for $i \geq 1$,

(2) $w_{i+1}=w_{i}$ implies $\left|w_{i}\right|=1$,

(3) there exists $c \in \mathbb{Z}$ such that $w_{i}=\overline{i+c}$ for all $i \gg 1$.

We call $c$ in (3) the charge of $w$. For example, the charge of $w=\cdots \overline{6} \overline{5} \overline{4} \overline{3} \overline{1} 2344 \in$ $\mathscr{F}$ is -3 . For each $i \in I$, we define the Kashiwara operators

$$
e_{i}, f_{i}: \mathscr{H} \longrightarrow \mathscr{F} \cup\{\mathbf{0}\}
$$

as in the case of $\mathcal{W}_{m \mid n}$ (see Section 2.2). They are well-defined since for $w=$ $\cdots w_{3} w_{2} w_{1} \in \mathscr{F}$ and $i \in I$,

$$
\epsilon^{(i)}(w)=\left(\cdots, \epsilon^{(i)}\left(w_{3}\right), \epsilon^{(i)}\left(w_{2}\right), \epsilon^{(i)}\left(w_{1}\right)\right)
$$


has only finitely many \pm 's (we read the signs from left to right). For $w \in \mathscr{F}$, we define

$$
\operatorname{wt}(w)=\Lambda+\sum_{b \in \mathbf{B}} m_{b} \epsilon_{b} \in P
$$

where $m_{b}=\left|\left\{k \mid w_{k}=b(k \geq 1)\right\}\right|-\delta_{|b| 0}$ for $b \in \mathbf{B}$. Since $m_{b}=0$ for almost all $b \in \mathbf{B}, \operatorname{wt}(w)$ is well-defined. For $w \in \mathscr{F}$ and $i \in I$, we set

$$
\varepsilon_{i}(w)=\max \left\{k \mid e_{i}^{k} w \neq \mathbf{0}\right\}, \quad \varphi_{i}(w)=\max \left\{k \mid f_{i}^{k} w \neq \mathbf{0}\right\} .
$$

For $c \in \mathbb{Z}$, let

$$
\begin{aligned}
H^{c} & =\cdots \overline{c+3} \overline{c+2} \overline{c+1}, & & \text { if } c \geq 0, \\
& =\cdots \overline{3} \overline{2} \overline{1} \underbrace{1 \cdots 1}_{|c|}, & & \text { if } c \leq 0 .
\end{aligned}
$$

Note that $\operatorname{wt}\left(H^{0}\right)=\Lambda$.

Proposition 5.1. $\mathscr{F}$ is a crystal graph for $\mathfrak{g}$, and

$$
\mathscr{H}=\bigoplus_{c \in \mathbb{Z}} \mathbf{B}(c)
$$

where $\mathbf{B}(c)$ is the connected component of $H^{c}(c \in \mathbb{Z})$. Moreover, $H^{c}$ is the highest weight element in $\mathscr{F}$, that is, $\operatorname{wt}\left(H^{c}\right) \geq \operatorname{wt}(w)$ for $w \in \mathbf{B}(c)$.

Proof: The conditions (b), (c) and (d) in Definition 2.1 are satisfied directly. So, it suffices to check that (a) holds. Given $w=\cdots w_{3} w_{2} w_{1} \in \mathscr{F}$ of charge $c$ and $i \in I$, choose a sufficiently large $M>0$ such that

(i) $w_{k}=\overline{c+k}$ for all $k \geq M$,

(ii) $\left\langle h_{i}, \operatorname{wt}\left(w^{>M}\right)\right\rangle=0$, where $w^{>M}=\cdots w_{M+2} w_{M+1} \in \mathscr{F}$.

Put $w^{\leq M}=w_{M} w_{M-1} \cdots w_{1}$. Then we may view $w^{\leq M}$ as an element in $\mathcal{W}_{m \mid n}$, where $m=M+c$ and $n \gg 0$. Also, we have $\varepsilon_{i}(w)=\varepsilon_{i}\left(w^{\leq M}\right), \varphi_{i}(w)=\varphi_{i}\left(w^{\leq M}\right)$, and $\left\langle h_{i}, \operatorname{wt}\left(w^{\leq M}\right)\right\rangle=\left\langle h_{i}, \operatorname{wt}(w)\right\rangle$. This implies the condition (a).

Since $w^{\leq M}$ is $\mathfrak{g l}_{m \mid n}$-equivalent to a semistandard tableau $P\left(w^{\leq M}\right)$ of a single column in $\mathbf{B}_{m \mid n}\left(\left(1^{M}\right)\right), w^{\leq M}$ is connected to the highest weight element $H_{m \mid n}^{\left(1^{M}\right)}$. If $P\left(w^{\leq M}\right)=f_{i_{1}} \cdots f_{i_{r}} H_{m \mid n}^{\left(1^{M}\right)}$ for some $r \geq 0$ and $i_{k} \in I_{m \mid n}(1 \leq k \leq r)$, then we have $w=f_{i_{1}} \ldots f_{i_{r}} H^{c}$. In particular, we have $\operatorname{wt}(w)=\operatorname{wt}\left(H^{c}\right)-\sum_{k=1}^{r} \alpha_{i_{k}} \leq \operatorname{wt}\left(H^{c}\right)$.

Let $x=\left\{x_{b} \mid b \in \mathbf{B}\right\}$. For $\mu \in P$, we define $x^{\mu}=\prod_{b \in \mathbf{B}} x_{b}^{m_{b}}$, where $\mu=k \Lambda+$ $\sum_{b \in \mathbf{B}} m_{b} \epsilon_{b}(k \in \mathbb{Z})$. Then the character of $\mathscr{F}$ is given by

$$
\operatorname{ch} \mathscr{F}=\sum_{w \in \mathscr{F}^{\prime}} x^{\mathrm{wt}(w)}=\frac{\prod_{b \in \mathbf{B}^{+}}\left(1+x_{b}^{-1}\right)}{\prod_{b^{\prime} \in \mathbf{B}^{-}}\left(1-x_{b^{\prime}}\right)} .
$$




\subsection{Semi-infinite semistandard tableaux for $\mathfrak{g}$}

Let us describe a crystal graph for $\mathfrak{g}$ occurring as a connected component in $\mathscr{F}^{\otimes u}$ $(u \geq 1)$. Let

$$
\mathbb{Z}_{+}^{u}=\left\{\lambda=\left(\lambda_{1}, \ldots, \lambda_{u}\right) \in \mathbb{Z}^{u} \mid \lambda_{1} \geq \lambda_{2} \geq \cdots \geq \lambda_{u}\right\}
$$

be the set of all generalized partitions of length $u$. For each $\lambda=\left(\lambda_{1}, \ldots, \lambda_{u}\right) \in \mathbb{Z}_{+}^{u}$, we call a $u$-tuple of semi-infinite words $\mathbf{w}=\left(w^{(1)}, \ldots, w^{(u)}\right) \in \mathscr{\mathscr { F }}^{\otimes u}$ a semistandard tableau of charge $\lambda$ if

(1) $w^{(i)}=\cdots w_{3}^{(i)} w_{2}^{(i)} w_{1}^{(i)} \in \mathscr{F}$, where the charge of $w^{(i)}$ is $\lambda_{i}$ for $1 \leq i \leq u$,

(2) $w_{k}^{(i)} \geq w_{k+d_{i}}^{(i+1)}$ for $1 \leq i<u$ and $k \geq 1$, where $d_{i}=\lambda_{i}-\lambda_{i+1}$,

(3) $w_{k}^{(i)}=w_{k+d_{i}}^{(i+1)}$ implies $\left|w_{k}^{(i)}\right|=0$.

We denote by $\mathbf{B}(\lambda)$ the set of all semistandard tableaux of charge $\lambda$. In fact, each $\mathbf{w} \in \mathbf{B}(\lambda)$ determines a unique semi-infinite tableau with infinitely many rows and $u$ columns, where each row of $\mathbf{w}$ reads (from left to right) as follows;

$$
w_{k+d_{1}+\cdots+d_{u-1}}^{(u)} \cdots w_{k+d_{1}+d_{2}}^{(3)} w_{k+d_{1}}^{(2)} w_{k}^{(1)}
$$

for $k \in \mathbb{Z}$ (we assume that $w_{k}^{(i)}$ is empty for $k \leq 0$ ).

Example 5.2. Let $\lambda=(3,1,-2,-2)$, and let $\mathbf{w}=\left(w^{(1)}, w^{(2)}, w^{(3)}, w^{(4)}\right)$ be given by

$\begin{array}{cccc}w^{(4)} & w^{(3)} & w^{(2)} & w^{(1)} \\ \vdots & \vdots & \vdots & \vdots \\ \overline{6} & \overline{6} & \overline{6} & \overline{6} \\ \overline{5} & \overline{5} & \overline{5} & \overline{5} \\ \overline{4} & \overline{4} & \overline{3} & \overline{1} \\ \overline{3} & \overline{3} & \overline{2} & \\ \overline{2} & \overline{2} & \overline{1} & \\ \overline{1} & \overline{1} & & \\ 1 & 2 & & \\ 1 & 3 & & \end{array}$.

Then $\mathbf{w}$ is a semistandard tableau of charge $\lambda$.

For $\lambda \in \mathbb{Z}_{+}^{u}$ and $\mathbf{w}=\left(w^{(1)}, \ldots, w^{(u)}\right) \in \mathbf{B}(\lambda)$, we may view $\mathbf{w}=w^{(1)} \otimes \cdots \otimes$ $w^{(u)} \in$

$\mathscr{F} \otimes u$, and consider $\mathbf{B}(\lambda)$ as a subset of $\mathscr{F} \otimes u$.

Proposition 5.3. For $\lambda \in \mathbb{Z}_{+}^{u}, \mathbf{B}(\lambda)$ together with $\mathbf{0}$ is stable under $e_{i}$ and $f_{i}(i \in I)$. Hence, $\mathbf{B}(\lambda)$ is a crystal graph for $\mathfrak{g}$. Furthermore, $\mathbf{B}(\lambda)$ is a connected I-colored oriented graph with a unique highest weight element $H^{\lambda}$. 
Proof: To show that $\mathbf{B}(\lambda)$ is a crystal graph for $\mathfrak{g}$, it is enough to check that $e_{i} \mathbf{w}, f_{i} \mathbf{w} \in$ $\mathbf{B}(\lambda) \cup\{\mathbf{0}\}$ for $\mathbf{w} \in \mathbf{B}(\lambda)$ and $i \in I$.

Suppose that $\mathbf{w} \in \mathbf{B}(\lambda)$ is given. Choose sufficiently large $m, n>0$. Then, for each $1 \leq i \leq u$, we have

$$
w^{(i)}=\cdots \overline{m+3} \overline{m+2} \overline{m+1} w_{*}^{(i)}=H^{m} w_{*}^{(i)}
$$

for some $w_{*}^{(i)} \in \mathcal{W}_{m \mid n}$. Set $\mathbf{w}_{*}=w_{*}^{(1)} \cdots w_{*}^{(u)} \in \mathcal{W}_{m \mid n}$. Then, $\mathbf{w}$ is $\mathfrak{g l}_{m \mid n}$-equivalent to $\mathbf{w}_{*}$. Note that $P\left(\mathbf{w}_{*}\right)$, the $P$-tableau of $\mathbf{w}_{*}$, is nothing but the $(m, n)$-hook semistandard tableau which is obtained from $\mathbf{w}$ by removing the entries smaller than $\bar{m}$. So, we have $e_{i} \mathbf{w}, f_{i} \mathbf{w} \in \mathbf{B}(\lambda) \cup\{\mathbf{0}\}$ for $i \in I_{m \mid n} \subset I$.

Now, consider $H^{\lambda}=\left(w^{(1)}, \ldots, w^{(u)}\right) \in \mathbf{B}(\lambda)$, where for $1 \leq i \leq u$,

$$
\begin{aligned}
w^{(i)} & =H^{\lambda_{i}}, & & \text { if } \lambda_{i} \geq 0, \\
& =H^{0} \underbrace{(u-i+1) \cdots(u-i+1)}_{\left|\lambda_{i}\right|}, & & \text { if } \lambda_{i}<0 .
\end{aligned}
$$

Since $P\left(\mathbf{w}_{*}\right)$ is connected to the highest weight element, it follows that $\mathbf{w}$ is connected to $H^{\lambda}$. Also, for $\mathbf{w} \in \mathbf{B}(\lambda)$, we have $\operatorname{wt}(w)=\sum_{i=1}^{r} \operatorname{wt}\left(w^{(i)}\right)$ and $\operatorname{wt}(\mathbf{w}) \leq \operatorname{wt}\left(H^{\lambda}\right)$. Hence, $\mathbf{B}(\lambda)$ is connected with the unique highest weight element $H^{\lambda}$.

Lemma 5.4. For $u \geq 1$, each connected component of $\mathscr{F} \otimes u$ is isomorphic to $\mathbf{B}(\lambda)$ for some $\lambda \in \mathbb{Z}_{+}^{u}$.

Proof: Suppose that $\mathbf{w}=w^{(1)} \otimes \cdots \otimes w^{(u)} \in \mathscr{F}^{\otimes u}$ is given. Choose sufficiently large $m, n>0$. Then, for each $1 \leq i \leq u$, we have $w^{(i)}=H^{m} w_{*}^{(i)}$, for some $w_{*}^{(i)} \in$ $\mathcal{W}_{m \mid n}$.

Set $\mathbf{w}_{*}=w_{*}^{(1)} \cdots w_{*}^{(u)} \in \mathcal{W}_{m \mid n}$. Then $\mathbf{w}$ is $\mathfrak{g l}_{m \mid n}$-equivalent to $\mathbf{w}_{*}$, and $P\left(\mathbf{w}_{*}\right) \in \mathbf{B}_{m \mid n}(\lambda)$ for some $\lambda=\left(\lambda_{k}\right)_{k \geq 1} \in \mathcal{P}_{m \mid n}$. We see that $\lambda_{1} \leq u$ from Schensted's algorithm.

For $1 \leq i \leq u$, let $w_{\sharp}^{(i)}$ be the word obtained by reading the $(u-i+1)^{\text {th }}$-column of $P\left(\mathbf{w}_{*}\right)$ from top to bottom (note that the columns in $\lambda$ are enumerated from left to right, but the words $w_{\sharp}^{(i)}(1 \leq i \leq u)$ are obtained from right to left). Set $\widetilde{\mathbf{w}}=$ $\widetilde{w}^{(1)} \otimes \cdots \otimes \widetilde{w}^{(u)} \in \mathscr{F}^{\otimes u}$ where $\widetilde{w}^{(i)}=H^{m} w_{\sharp}^{(i)}$. Then $\widetilde{\mathbf{w}}$ is $\mathfrak{g l}_{m \mid n}$-equivalent to $\mathbf{w}$. Also, $\widetilde{\mathbf{w}}$ is uniquely determined independent of all sufficiently large $m, n$, and hence it is $\mathfrak{g}$-equivalent to $\mathbf{w}$.

Let $\mu_{i}$ be the charge of $\widetilde{w}^{(i)}(1 \leq i \leq u)$. Since the length of $w_{\sharp}^{(i)}$ is less than or equal to that of $w_{\sharp}^{(i+1)}$, we have $\mu_{i} \geq \mu_{i+1}$ for $1 \leq i \leq u-1$, and hence $\widetilde{\mathbf{w}}$ is a semistandard tableau of charge $\mu$, where $\mu=\left(\mu_{1}, \ldots, \mu_{u}\right) \in \mathbb{Z}_{+}^{u}$.

By Lemma 2.9, we can also check the following lemma, which implies that each $\mathbf{w} \in \mathscr{F}^{\otimes u}$ is $\mathfrak{g}$-equivalent to a unique semi-infinite semistandard tableau.

Lemma 5.5. Let $\mathbf{w}$ and $\mathbf{w}^{\prime}$ be two semi-infinite semistandard tableaux. If $\mathbf{w} \simeq_{\mathfrak{g}} \mathbf{w}^{\prime}$, then $\mathbf{w}=\mathbf{w}^{\prime}$. 


\subsection{Rational semistandard tableaux for $\mathfrak{g l}_{u}$}

Let us recall the crystal graphs of rational representations of $\mathfrak{g l}_{u \mid 0}$ for $u \geq 2$. By convention, we write $\mathfrak{g l}_{u}=\mathfrak{g l}_{u \mid 0}, \mathbf{B}_{u}=\mathbf{B}_{u \mid 0}, I_{u}=I_{u \mid 0}, P_{u}=P_{u \mid 0}$ and so on.

Let $\mathbf{B}_{u}^{\vee}=\{-\overline{1}<-\overline{2}<\cdots<-\bar{u}\}$ be the dual crystal graph of $\mathbf{B}_{u}$ whose associated graph is given by

$$
-\overline{1} \stackrel{\overline{1}}{\longrightarrow}-\overline{2} \stackrel{\overline{2}}{\longrightarrow} \cdots \stackrel{\overline{u-1}}{\longrightarrow}-\bar{u}
$$

where $\operatorname{wt}(-\bar{k})=-\operatorname{wt}(\bar{k})=-\epsilon_{\bar{k}}$ for $1 \leq k \leq u$ (cf. [16]).

Given $\lambda=\left(\lambda_{1}, \ldots, \lambda_{u}\right) \in \mathbb{Z}_{+}^{u}$, we may identify $\lambda$ with a generalized Young diagram in the following way. First, we fix a vertical line. Then for each $\lambda_{k}$, we place $\left|\lambda_{k}\right|$ nodes (or boxes) in the $k^{\text {th }}$ row in a left-justified (resp. right-justified) way with respect to the vertical line if $\lambda_{k} \geq 0$ (resp. $\lambda_{k} \leq 0$ ). For example,

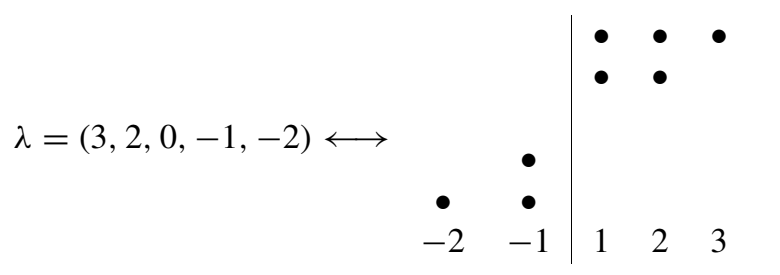

We enumerate the columns of a diagram as in the above figure.

Definition 5.6 (cf. [20]). Let $T$ be a tableau obtained by filling a generalized Young diagram $\lambda$ of length $u$ with the entries in $\mathbf{B}_{u} \cup \mathbf{B}_{u}^{\vee}$. We call $T$ a rational semistandard of shape $\lambda$ if

(1) the entries in the columns indexed by positive (resp. negative) numbers belong to $\mathbf{B}_{u}\left(\operatorname{resp} . \mathbf{B}_{u}^{\vee}\right)$

(2) the entries in each row (resp. column) are weakly (resp. strictly) increasing from left to right (resp. from top to bottom),

(3) if $b_{1}<\cdots<b_{s}$ (resp. $-b_{1}^{\prime}<\cdots<-b_{t}^{\prime}$ ) are the entries in the $1^{\text {st }}$ (resp. $-1^{\text {st }}$ ) column $(s+t \leq u)$, then

$$
b_{i}^{\prime \prime} \leq b_{i}
$$

for $1 \leq i \leq s$, where $\left\{b_{1}^{\prime \prime}<\cdots<b_{u-t}^{\prime \prime}\right\}=\mathbf{B}_{u} \backslash\left\{b_{1}^{\prime}, \ldots, b_{t}^{\prime}\right\}$

We denote by $\mathbf{B}_{u}(\lambda)$ the set of all rational semistandard tableaux of shape $\lambda$. 
Example 5.7. For $\lambda=(3,2,0,-1,-2)$, we have

$$
\begin{aligned}
& \begin{array}{lll|lll}
\overline{4} & \overline{3} & \overline{1} & \overline{4} & \overline{3} & \overline{1} \\
\overline{2} & \overline{2} & & \overline{2} & \overline{2} &
\end{array} \\
& \in \mathbf{B}_{5}(\lambda) \text {, but } \\
& \notin \mathbf{B}_{5}(\lambda) \text {. } \\
& \begin{array}{rr|rr} 
& -\overline{1} & -\overline{4} \\
-\overline{2} & -\overline{2} & -\overline{2} & -\overline{5}
\end{array}
\end{aligned}
$$

Let us explain the relation between the crystal graphs of rational representations and polynomial representations. Let $T$ be a rational semistandard tableau in $\mathbf{B}_{u}\left((-1)^{t}\right)(0 \leq$ $t \leq u)$ with the entries $-b_{1}<\cdots<-b_{t}$. We define $\sigma(T)$ be the tableau in $\mathbf{B}_{u}\left(1^{u-t}\right)$ with the entries $b_{1}^{\prime}<\cdots<b_{u-t}^{\prime}$, where $\left\{b_{1}^{\prime}<\cdots<b_{u-t}^{\prime}\right\}=\mathbf{B}_{u} \backslash\left\{b_{1}<\cdots<b_{t}\right\}$. If $t=u$, then we define $\sigma(T)$ to be the empty word.

Generally, for $\lambda \in \mathbb{Z}_{+}^{u}$ and $T \in \mathbf{B}_{u}(\lambda)$, we define $\sigma(T)$ to be the tableau obtained by applying $\sigma$ to the $-1^{\text {st }}$ column of $T$. For example, when $u=5$, we have

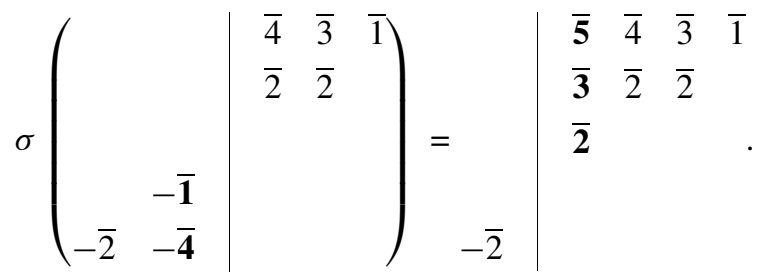

Now, we embed $\mathbf{B}_{u}(\lambda)$ into $\left(\mathbf{B}_{u} \oplus \mathbf{B}_{u}^{\vee}\right)^{\otimes N}\left(N=\sum_{k \geq 1}\left|\lambda_{k}\right|\right)$ by column reading of tableaux (cf. Section 2.2), and apply $e_{i}, f_{i}$ to $\mathbf{B}_{u}(\lambda)\left(i \in I_{u}\right)$. Then

Lemma 5.8. For $\lambda=\left(\lambda_{1}, \ldots, \lambda_{u}\right) \in \mathbb{Z}_{+}^{u}, \mathbf{B}_{u}(\lambda)$ is a crystal graph for $\mathfrak{g l}_{u}$, and the map

$$
\sigma: \mathbf{B}_{u}(\lambda) \cup\{\mathbf{0}\} \rightarrow \mathbf{B}_{u}\left(\lambda+\left(1^{u}\right)\right) \cup\{\mathbf{0}\},
$$

$(\sigma(\mathbf{0})=\mathbf{0})$ is a bijection which commutes with $e_{i}, f_{i}$ for $i \in I_{u}$, where $\operatorname{wt}(\sigma(T))=$ $\operatorname{wt}(T)+\left(\epsilon_{\bar{u}}+\cdots+\epsilon_{\overline{1}}\right)$ for $T \in \mathbf{B}_{u}(\lambda)$.

Proof: By Definition 5.6, it is straightforward to check that $\sigma(T) \in \mathbf{B}_{u}\left(\lambda+\left(1^{u}\right)\right)$, where we define $\mu+v=\left(\mu_{k}+v_{k}\right)_{k \geq 1}$ for two generalized partitions $\mu=\left(\mu_{k}\right)_{k \geq 1}$ and $v=\left(v_{k}\right)_{\geq 1}$ in $\mathbb{Z}_{+}^{u}$. It follows immediately that $\sigma$ is a bijection and $\operatorname{wt}(\sigma(T))=$ $\operatorname{wt}(T)+\left(\epsilon_{\bar{u}}+\cdots+\epsilon_{\overline{1}}\right)$ for $T \in \mathbf{B}_{u}(\lambda)$.

So, it remains to show that $\mathbf{B}_{u}(\lambda)$ is a crystal graph for $\mathfrak{g l}_{u}$, and $\sigma$ commutes with the Kashiwara operators.

The claim follows easily when $\lambda_{i} \geq 0$ for all $1 \leq i \leq n$. Next, consider $\lambda=\left((-1)^{t}\right)$ for some $0 \leq t \leq u$. Then it is also straightforward to check that our claim holds. Now, for a general $\lambda \in \mathbb{Z}_{+}^{n}$, any tableau $T$ in $\mathbf{B}_{u}(\lambda)$ can be viewed as a tensor product of its columns when we apply $e_{i}, f_{i}$. By definition of tensor product of crystal graphs (Definition 2.3) and the argument in the case of $\lambda=\left((-1)^{t}\right)$, we have $\sigma\left(x_{i} T\right)=x_{i} \sigma(T)$ for $x=e, f$ and $i \in I_{u}$. So we have $x_{i} \sigma(T) \in \mathbf{B}_{u}\left(\lambda+\left(1^{u}\right)\right)$ if and only if $x_{i} T \in \mathbf{B}_{u}(\lambda)$. Springer 
So, our claim follows by induction on the number of columns indexed by negative numbers.

Note that for $\lambda \in \mathbb{Z}_{+}^{u}$, there exists a unique highest weight element $H_{u}^{\lambda}$ in $\mathbf{B}_{u}(\lambda)$. In fact, $H_{u}^{\lambda}=\sigma^{-k}\left(H_{u}^{\lambda+\left(k^{u}\right)}\right)$ for all $k \geq 0$ such that $\lambda+\left(k^{u}\right)$ is an ordinary partition.

Let $y=\left\{y_{b} \mid b \in \mathbf{B}_{u}\right\}$ be the set of variables indexed by $\mathbf{B}_{u}$. For $\mu=\sum_{b \in \mathbf{B}_{u}} \mu_{b} \epsilon_{b} \in$ $P_{u}$, we set $y^{\mu}=\prod_{b \in \mathbf{B}_{u}} y_{b}^{\mu_{b}}$. For $\lambda \in \mathbb{Z}_{+}^{u}$, the character of $\mathbf{B}_{u}(\lambda)$ is given by a rational Schur function corresponding to $\lambda$;

$$
s_{\lambda}(y)=\sum_{T \in \mathbf{B}_{u}(\lambda)} y^{\mathrm{wt} T} .
$$

By Lemma 5.8, we have $s_{\lambda+\left(1^{u}\right)}(y)=\left(y_{\bar{u}} \cdots y_{\overline{1}}\right) s_{\lambda}(y)$.

\subsection{Decomposition of $\mathscr{F} \otimes u$}

Now, let us decompose $\mathscr{F}^{\otimes u}$ for $u \geq 2$. We start with another description of $\mathscr{F}^{\otimes u}$ in terms of matrices of non-negative integers. Set

$$
\begin{aligned}
\mathscr{M}^{u}= & \left\{A=\left(a_{b b^{\prime}}\right)_{b \in \mathbf{B}, b^{\prime} \in \mathbf{B}_{u}} \mid\right. \\
& \text { (1) } a_{b b^{\prime}} \in \mathbb{Z}_{\geq 0}, \\
& \text { (2) } a_{b b^{\prime}} \leq 1 \text { if }|b|=0, \\
& \text { (3) } \left.a_{b b^{\prime}}=1 \text { for all } b \ll \overline{1} \text {, and } a_{b b^{\prime}}=0 \text { for all } b \gg 1\right\} .
\end{aligned}
$$

For $\mathbf{w}=w^{(1)} \otimes \cdots \otimes w^{(u)} \in \mathscr{F}^{\otimes u}$, set $A(\mathbf{w})=\left(a_{b \bar{k}}\right) \in \mathscr{M}^{u}$, where $a_{b \bar{k}}$ is the number of occurrences of $b$ in $w^{(u-k+1)}(1 \leq k \leq u)$. Then the map $\mathbf{w} \mapsto A(\mathbf{w})$ is a bijection from $\mathscr{F}^{\otimes u}$ to $\mathscr{M}^{u}$, where each $w^{(i)}$ corresponds to the $i^{\text {th }}$-column of $A(\mathbf{w})$ for $1 \leq i \leq u$.

\section{Example 5.9.}

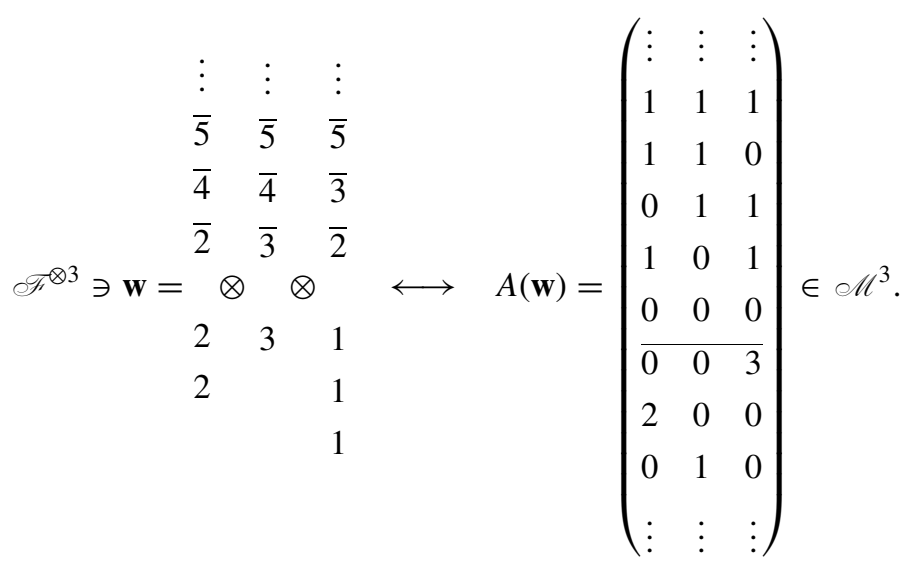


Let us define the crystal graph structures on $\mathscr{C}^{u}$, which are naturally induced from those on $\mathbf{M}_{m|n, u| 0}^{\sharp}$ in Section 4. For $m, n>0$, we define

$$
\operatorname{Res}_{m \mid n}: \mathscr{M}^{u} \longrightarrow \mathbf{M}_{m|n, u| 0}^{\sharp},
$$

by $\operatorname{Res}_{m \mid n} A=\left(a_{b b^{\prime}}\right)_{b \in \mathbf{B}_{m \mid n}, b^{\prime} \in \mathbf{B}_{u}}$ for $A=\left(a_{b b^{\prime}}\right) \in \mathscr{M}^{u}$.

For $A \in \mathscr{M}^{u}$ and $i \in I$, we define $e_{i} A$ and $f_{i} A$ to be the unique elements in $\mathscr{M}^{u} \cup\{\mathbf{0}\}$ satisfying

$$
\operatorname{Res}_{m \mid n}\left(e_{i} A\right)=e_{i}\left(\operatorname{Res}_{m \mid n} A\right), \operatorname{Res}_{m \mid n}\left(f_{i} A\right)=f_{i}\left(\operatorname{Res}_{m \mid n} A\right),
$$

for all sufficiently large $m, n>0$, where we assume that $\operatorname{Res}_{m \mid n} \mathbf{0}=\mathbf{0}$. We set

$$
\begin{aligned}
\operatorname{wt}(A) & =u \Lambda+\sum_{b \in \mathbf{B}} m_{b} \epsilon_{b}, \\
\varepsilon_{i}(A) & =\max \left\{k \mid e_{i}^{k} A \neq \mathbf{0}\right\}, \quad \varphi_{i}(A)=\max \left\{k \mid f_{i}^{k} A \neq \mathbf{0}\right\},
\end{aligned}
$$

for $A \in \mathscr{M}^{u}$ and $i \in I$, where $m_{b}=\sum_{b^{\prime} \in \mathbf{B}_{u}}\left(a_{b b^{\prime}}-\delta_{|b| 0}\right)$. Then it is not difficult to check the following lemma;

Lemma 5.10. $\mathscr{C M}^{u}$ is a crystal graph for $\mathfrak{g}$, and the map $\mathbf{w} \mapsto A(\mathbf{w})$ is an isomorphism of $\mathfrak{g}$-crystals from $\mathscr{F}^{\otimes u}$ to $\mathscr{C}^{u}$. In particular, for $A \in \mathscr{M}^{\mu}$, A is $\mathfrak{g}$-equivalent to a unique semistandard tableau in $\mathbf{B}(\lambda)$ for some $\lambda \in \mathbb{Z}_{+}^{u}$.

Next, let us define a $\mathfrak{g l}_{u}$-crystal structure on $\mathscr{M}^{u}$. Since $\mathscr{M}^{u}$ can be identified with $\mathscr{F}^{\otimes u}$, this induces a $\mathfrak{g l}_{u}$-crystal structure on $\mathscr{F}^{\otimes u}$. For $A \in \mathscr{M}^{u}$ and $j \in I_{u}$, we define $e_{j}^{*} A$ and $f_{j}^{*} A$ to be the unique elements in $\mathscr{M} \cup\{\mathbf{0}\}$ satisfying

$$
\operatorname{Res}_{m \mid n}\left(e_{j}^{*} A\right)=e_{j}^{*}\left(\operatorname{Res}_{m \mid n} A\right), \operatorname{Res}_{m \mid n}\left(f_{j}^{*} A\right)=f_{j}^{*}\left(\operatorname{Res}_{m \mid n} A\right),
$$

for all sufficiently large $m, n>0$. We set

$$
\begin{aligned}
\mathrm{wt}^{*}(A) & =\sum_{b \in \mathbf{B}_{u}} m_{b} \epsilon_{b}, \\
\varepsilon_{j}^{*}(A) & =\max \left\{k \mid\left(e_{j}^{*}\right)^{k} A \neq \mathbf{0}\right\}, \quad \varphi_{j}^{*}(A)=\max \left\{k \mid\left(f_{j}^{*}\right)^{k} A \neq \mathbf{0}\right\},
\end{aligned}
$$

for $A \in \mathscr{M}^{u}$ and $j \in I_{u}$, where $m_{b}=\sum_{b^{\prime} \in \mathbf{B}}\left(a_{b^{\prime} b}-\delta_{\left|b^{\prime}\right| 0}\right)$. Then

Lemma 5.11. $\mathscr{M}^{u}$ is a crystal graph for $\mathfrak{g l}_{u}$ with respect to $e_{j}^{*}, f_{j}^{*}\left(j \in I_{u}\right)$, and each $A \in \mathscr{M}^{u}$ is $\mathfrak{g l}_{u}$-equivalent to a unique rational semistandard tableau.

Proof: It is clear that $\mathscr{C}^{u}$ is a crystal graph for $\mathfrak{g l}_{u}$. For $A \in \mathscr{C}^{u}$, choose sufficiently large $m, n$. As an element in a $\mathfrak{g l}_{u}$-crystal, consider a semistandard tableau $T=P_{2}^{\sharp}\left(\operatorname{Res}_{m \mid n} A\right)($ see $(4.6))$. We observe that $\operatorname{wt}(T)=\mathrm{wt}^{*}(A)+k\left(\epsilon_{\bar{u}}+\cdots+\epsilon_{\overline{1}}\right)$ Springer 
for some $k \geq 0$. Then, by Lemma 5.8, the rational semistandard tableau $\sigma^{-k}(T)$, say $S$, is $\mathfrak{g l}_{u}$-equivalent to $A$. Note that $S$ is independent of $m$ and $n$ since wt* $(A)$ is fixed.

Suppose that $A \in \mathscr{M}^{u}$ is given. By Lemma 5.10, there exists a unique semistandard tableau $\mathbf{w}$ in $\mathbf{B}(\lambda)$ for some $\lambda \in \mathbb{Z}_{+}^{u}$, which is $\mathfrak{g}$-equivalent to $A$, and we set $\mathscr{P}_{1}(A)=\mathbf{w}$. Also, by Lemma 5.11, there exists a unique rational semistandard tableau $T$, which is $\mathfrak{g l}_{u}$-equivalent to $A$, and we set $\mathscr{P}_{2}(A)=T$. Now, we define

$$
\varpi(A)=\left(\mathscr{P}_{1}(A), \mathscr{P}_{2}(A)\right)
$$

Proposition 5.12. $\mathscr{M}^{\mu}$ is a $\left(\mathfrak{g}, \mathfrak{g l}_{u}\right)$-bicrystal, and for each connected component $\mathscr{C}$ in $\mathscr{M}^{u}$, $\varpi$ gives the following isomorphism of $\left(\mathfrak{g}, \mathfrak{g l}_{u}\right)$-bicrystals;

$$
\varpi: \mathscr{C} \longrightarrow \mathbf{B}(\lambda) \times \mathbf{B}_{u}(\mu),
$$

for some $\lambda, \mu \in \mathbb{Z}_{+}^{u}$.

Proof: It follows from Proposition 4.2.

Example 5.13. Let $A$ be given in Example 5.9. Then

$$
\begin{aligned}
& \vdots \quad \vdots \quad \vdots \quad \vdots \vdots \vdots \\
& \begin{array}{llll}
\overline{5} & \overline{5} & \overline{5} & \overline{5} \\
\hline & \overline{5}
\end{array} \\
& \begin{array}{llll}
\overline{4} & \overline{4} & \overline{3} & \overline{4} \\
4 & \overline{2}
\end{array}
\end{aligned}
$$

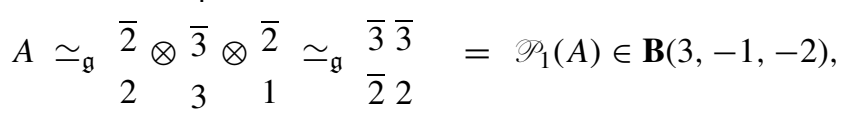

$$
\begin{aligned}
& 2 \quad 1 \quad 12 \\
& 1 \quad 13 \\
& 1
\end{aligned}
$$

On the other hand, consider $\operatorname{Res}_{4 \mid 3} A$. Then

$$
\begin{aligned}
& \operatorname{Res}_{4 \mid 3} A \quad \simeq_{\mathfrak{g l}_{u}} \overline{2} \otimes \overline{3} \overline{3} \otimes \overline{1} \overline{1} \overline{1} \otimes \frac{\overline{3}}{1} \otimes \frac{\overline{2}}{\overline{1}} \otimes \frac{\overline{3}}{\overline{2}} \\
& \begin{array}{llllll}
\overline{3} & \overline{3} & \overline{3} & \overline{3} & \overline{2} & \overline{1}
\end{array}
\end{aligned}
$$

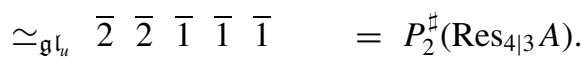


Since $\operatorname{wt}(A)=-\epsilon_{\overline{2}}+\epsilon_{\overline{1}}$, we have

$$
\mathscr{P}_{2}(A)=\sigma^{-4}\left(P_{2}^{\sharp}\left(\operatorname{Res}_{4 \mid 3} A\right)\right)=\quad \mid \begin{aligned}
& \overline{2} \overline{1} \\
& \overline{1}
\end{aligned} \in \mathbf{B}_{3}(2,1,-3) .
$$

Now, we let

$$
\mathscr{M}_{\text {h.w. }}^{u}=\left\{A \mid \varpi(A)=\left(H^{\lambda}, H_{u}^{\mu}\right) \text { for some } \lambda, \mu \in \mathbb{Z}_{+}^{u}\right\}
$$

be the set of all the highest weight elements in $\mathscr{M}^{\mu}$. Then $\mathscr{M}^{u}$ is the direct sum of the connected components of the elements in $\mathscr{M}_{\text {h.w. }}^{u}$ as a $\left(\mathfrak{g}, \mathfrak{g l}_{u}\right)$-bicrystal. For $\lambda \in \mathbb{Z}_{+}^{u}$, let $\mu_{i}(1 \leq i \leq u)$ be the number of occurrences of $i$ in $H^{\lambda}$. Put $\nu_{i}=\mu_{i}-\mu_{i+1}$ for $1 \leq i \leq u$ where $\mu_{u+1}=0$. Then we define $\mathscr{A}_{\lambda}=\left(a_{b b^{\prime}}\right) \in \mathscr{M}^{\mu}$ by

(1) for $k \geq 1,0 \leq l<u$,

$$
a_{\bar{k} \overline{u-l}}= \begin{cases}1, & \text { if there exists } \bar{k} \text { in the }(l+1)^{\mathrm{th}}-\text { column of } H^{\lambda} \\ 0, & \text { otherwise }\end{cases}
$$

(2) for $k \geq 1,0 \leq l<u$,

$$
a_{k \overline{u-l}}= \begin{cases}v_{k+l}, & \text { if } 1 \leq k+l \leq u \\ 0, & \text { otherwise }\end{cases}
$$

(cf. (4.8)). Then, we can check that $\varpi\left(\mathscr{C}_{\lambda}\right)=\left(H^{\lambda}, H_{u}^{\lambda^{*}}\right)$, where

$$
\lambda^{*}=\left(-\lambda_{u}, \ldots,-\lambda_{1}\right) \in \mathbb{Z}_{+}^{u} .
$$

Theorem 5.14. We have

$$
\mathscr{M}_{\text {h.w. }}^{u}=\left\{\mathscr{A}_{\lambda} \mid \lambda \in \mathbb{Z}_{+}^{u}\right\}
$$

and the following isomorphism of $\left(\mathfrak{g}, \mathfrak{g l}_{u}\right)$-bicrystals;

$$
\varpi: \mathscr{M}^{u} \longrightarrow \bigoplus_{\lambda \in \mathbb{Z}_{+}^{u}} \mathbf{B}(\lambda) \times \mathbf{B}_{u}\left(\lambda^{*}\right)
$$

Proof: Let $A$ be a highest weight element in $\mathscr{M}^{u}$. Then, $\operatorname{Res}_{m \mid n} A$ is a highest weight element in $\mathbf{M}_{m|n, u| 0}^{\sharp}$ for all sufficiently large $m, n>0$. By Theorem 4.5, it follows that $A=\mathscr{A}_{\lambda}$ for some $\lambda \in \mathbb{Z}_{+}^{u}$. 
The character of $\mathscr{M}^{u}$ is given by

$$
\operatorname{ch} \odot \mathscr{U}^{u}=\sum_{A \in M^{u}} x^{\mathrm{wt}(A)} y^{\mathrm{wt}^{*}(A)}=\frac{\prod_{b \in \mathbf{B}^{+}} \prod_{b^{\prime} \in \mathbf{B}_{u}}\left(1+x_{b}^{-1} y_{b^{\prime}}^{-1}\right)}{\prod_{b \in \mathbf{B}^{-}} \prod_{b^{\prime} \in \mathbf{B}_{u}}\left(1-x_{b} y_{b^{\prime}}\right)} .
$$

By Theorem 5.14, we obtain the following identity;

$$
\frac{\prod_{b \in \mathbf{B}^{+}} \prod_{b^{\prime} \in \mathbf{B}_{u}}\left(1+x_{b}^{-1} y_{b^{\prime}}^{-1}\right)}{\prod_{b \in \mathbf{B}^{-}} \prod_{b^{\prime} \in \mathbf{B}_{u}}\left(1-x_{b} y_{b^{\prime}}\right)}=\sum_{\lambda \in \mathbb{Z}_{+}^{u}} \operatorname{ch} \mathbf{B}(\lambda) s_{\lambda^{*}}(y),
$$

where $\operatorname{ch} \mathbf{B}(\lambda)=\sum_{\mathbf{w} \in \mathbf{B}(\lambda)} x^{\mathrm{wt}(\mathbf{w})}$ is the character of $\mathbf{B}(\lambda)$, and $s_{\lambda^{*}}(y)$ is the rational Schur function corresponding to $\lambda^{*}$.

From the classical Cauchy identities of Schur functions (cf. [18]), it follows that the left hand side of (5.5) is equal to

$$
\sum_{\mu, v \in \mathcal{P}} s_{\mu}\left(y^{-1}\right) s_{\mu^{\prime}}\left(x_{+}^{-1}\right) s_{v}(y) s_{v}\left(x_{-}\right)
$$

where $\mathcal{P}$ is the set of all partitions, $y^{-1}=\left\{y_{b}^{-1} \mid b \in \mathbf{B}_{u}\right\}, x_{+}^{-1}=\left\{x_{b}^{-1} \mid b \in \mathbf{B}^{+}\right\}$, and $x_{-}=\left\{x_{b} \mid b \in \mathbf{B}^{-}\right\}$. Note that $s_{\mu}\left(y^{-1}\right)=s_{\mu^{*}}(y)$ and

$$
s_{\mu^{*}}(y) s_{\nu}(y)=\sum_{\lambda \in \mathbb{Z}_{+}^{u}} N_{\mu \nu}^{\lambda} s_{\lambda}(y)
$$

for some $N_{\mu \nu}^{\lambda} \in \mathbb{Z}_{\geq 0}$. Then the left hand side of (5.5) can be written as

$$
\sum_{\lambda \in \mathbb{Z}_{+}^{u}}\left(\sum_{\mu, v \in \mathcal{P}} N_{\mu \nu}^{\lambda} s_{\mu^{\prime}}\left(x_{+}^{-1}\right) s_{v}\left(x_{-}\right)\right) s_{\lambda}(y)
$$

From a linear independence of $\left\{s_{v}(y) \mid v \in \mathbb{Z}_{+}^{u}\right\}$ (see Lemma 3.1 [3]), we obtain the following character formula of $\mathbf{B}(\lambda)$ by comparing with the right hand side of (5.5);

Corollary 5.15 (cf. $[3,13])$. For $\lambda \in \mathbb{Z}_{+}^{u}$, we have

$$
\operatorname{ch} \mathbf{B}(\lambda)=\sum_{\mu, \nu \in \mathcal{P}} N_{\mu \nu^{\prime}}^{\lambda^{*}} s_{\mu^{\prime}}\left(x_{+}^{-1}\right) s_{\nu}\left(x_{-}\right)
$$

Remark 5.16. Using Howe duality [9], there have been many works on the multiplicity free decomposition of Fock space representations with respect to an action of a pair of Lie (super)algebras, usually called a dual pair (for example, see [8] for the dual pairs of affine Kac-Moody algebras, [13] for the dual pair $\left(\widehat{\mathfrak{g l}}_{\infty}, \mathfrak{g l}_{n}\right)$, and [5] for the dual pair $\left.\left(\widehat{\mathfrak{g l}}_{\infty \mid \infty}, \mathfrak{g l}_{n}\right)\right)$. We may view Theorem 5.14 as a crystal version of the Howe duality of $\left(\mathfrak{g}, \mathfrak{g l}_{u}\right)$, and also expect similar combinatorial analogues for other dual pairs acting on Fock space representations. 
Acknowledgements The author would like to thank Prof. S.-J. Kang and Prof. S.-J. Cheng for their interests in this work and many helpful discussions.

\section{References}

1. G. Benkart, S.-J. Kang, and M. Kashiwara, "Crystal bases for the quantum superalgebra $U_{q}(\mathfrak{g l}(m, n))$," J. Amer. Math. Soc. 13(2) (2000), 295-331.

2. A. Berele and A. Regev, "Hook Young diagrams with applications to combinatorics and to the representations of Lie superalgebras," Adv. Math. 64 (1987), 118-175.

3. S.-J. Cheng and N. Lam, "Infinite-dimensional Lie superalgebras and hook Schur functions," Comm. Math. Phys. 238(1-2) (2003), 95-118.

4. S.-J. Cheng and W. Wang, "Howe duality for Lie superalgebras," Compositio Math. 128(1) (2001), 55-94.

5. S.-J. Cheng and W. Wang, "Lie subalgebras of differential operators on the super circle," Publ. Res. Inst. Math. Sci. 39(3) (2003), 545-600.

6. V.I. Danilov and G.A. Koshevoy, "Bi-crystals and crystal $(G L(V), G L(W))$ duality," RIMS preprint, (2004) No. 1458.

7. W. Fulton, "Young tableaux," London Mathematical Society Student Texts, Vol. 35 Cambridge University Press," Cambridge, 1997.

8. K. Hasegawa, "Spin module versions of Weyl's reciprocity theorem for classical Kac-Moody Lie algebras - an application to branching rule duality," Publ. Res. Inst. Math. Sci. 25(5) (1989), 741-828.

9. R. Howe, "Remarks on classical invariant theory," Trans. Amer. Math. Soc. 313(2) (1989), 539-570.

10. V.G. Kac, Infinite Dimensional Lie Algebras. Cambridge University Press, 3rd ed., 1990.

11. V.G. Kac, "Lie superalgebras," Adv. in Math. 26(1) (1977), 8-96.

12. V.G. Kac and J.W. van de Leur, "Super boson-fermion correspondence," Ann. Inst. Fourier 37(4) (1987), 99-137.

13. V. G. Kac and A. Radul, "Representation theory of the vertex algebra $W_{1+\infty}$," Transform. Groups 1(1-2) (1996), 41-70.

14. S.-J. Kang and J.-H. Kwon, "Tensor product of crystal bases for $U_{q}(\mathfrak{g l}(m, n))$-modules," Comm. Math. Phys. 224 (2001), 705-732.

15. M. Kashiwara, "Crystal bases and Littelmann's refined Demazure character formula," Duke Math. J. 71 (1993), 839-858.

16. M. Kashiwara, "On crystal bases," Representations of groups, in CMS Conf. Proc., Vol. 16, Amer. Math. Soc., Providence, RI, (1995), pp. 155-197.

17. D. Knuth, "Permutations, matrices, and the generalized Young tableaux," Pacific J. Math. 34 (1970), 709-727.

18. I.G. Macdonald, "Symmetric functions and Hall polynomials," Oxford University Press, 2nd ed., 1995.

19. J.B. Remmel, "The combinatorics of ( $k, l$ )-hook Schur functions," Contemp. Math. 34 (1984), $253-287$.

20. J.R. Stembridge, "Rational tableaux and the tensor algebra of $\mathfrak{g l}_{n}$," J. Combin. Theory Ser. A 46(1) (1987), 79-120. 